\title{
Desempeño relativo de la productividad física de la ganadería de Nueva Zelanda y Uruguay, 1870-2010
}

\author{
Jorge ÁLVAREZ SCANNIELLO
}

PALABRAS CLAVE: economías de nuevo asentamiento europeo, productividad ganadera, convergencia-divergencia, trayectoria tecnológica.

\section{CÓDIGOS JEL: N56, N57, O13, 033.}

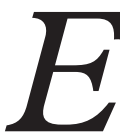

n el siglo XIX, Nueva Zelanda y Uruguay fueron sociedades de nuevo asentamiento europeo, ricas en recursos naturales, que se insertaron en la economía mundial como productoras y exportadoras de bienes ganaderos, pero que mostraron desempeños divergentes a largo plazo.

El objetivo del artículo es identificar las principales tendencias históricas del desempeño relativo de la productividad física de la ganadería de ambos países y comprender los procesos de convergencia-divergencia, a la luz del concepto de trayectoria tecnológica. Para ello, se realiza una revisión de los indicadores sintéticos unidad ganadera y carne equivalente, se proponen correcciones a los métodos de agregación usuales, teniendo en cuenta las especificidades de cada sistema ganadero, y se comparan las trayectorias tecnológicas involucradas en el crecimiento de la productividad de la ganadería. 


\title{
Comparative physical productivity of livestock in New Zealand and Uruguay, 1870-2010
}

\section{KEYWORDS: European settler societies, livestock productivity, con- vergence-divergence, technological trajectory.}

\author{
JEL CODES: N56, N57, O13, 033.
}

the nineteenth century, New Zealand and Uruguay were new European set-
tler societies and rich in natural resources. Both countries engaged with the
world economy as producers and exporters of livestock products. However, these countries exhibited divergent long-term development.

The aim of this article is to identify the main historical tendencies associated with the relative physical productivity of livestock in both countries and to understand convergent-divergent processes in light of their technological trajectories. The main synthetic indicators used in agrarian sciences, such as Livestock Units and Meat Equivalents, are reviewed and a series of corrections that account for the specific features of each agrarian system is proposed for the usual aggregation methods. The technological trajectories that affected livestock productivity growth are also compared.

The main results indicate that Uruguayan livestock production fell behind that of New Zealand due to the technological trajectory followed by each country. In the nineteenth century, Uruguay had very favourable natural conditions for livestock production. It did not develop technologies to improve soil and land productivity until the second half of the twentieth century. In contrast, New Zealand began to improve grassland productivity during the nineteenth century in order to increase and diversify livestock production.

Recepción: 2018-10-31 - Revisión: 2019-05-21 - Aceptación: 2019-07-04

Jorge Álvarez Scanniello [orcid.org/0000-0003-4135-9784] es profesor del Programa de Historia Económica y Social, Unidad Multidisciplinaria, de la Facultad de Ciencias Sociales de la Universidad de la República, Uruguay, e investigador nivel 1 del Sistema Nacional de Investigadores. Dirección para correspondencia: Constituyente 1502, CP 11200, Facultad de Ciencias Sociales, UdelaR, 4. ${ }^{\circ}$ piso, Unidad Multidisciplinaria, Montevideo (Uruguay).C.e.:jorge.alvarez@cienciassociales.edu.uy 


\section{INTRODUCCIÓN}

Desde el siglo XIX, Nueva Zelanda y Uruguay fueron economías de nuevo asentamiento europeo (Denoon, 1983; Lloyd, Metzer \& Sutch, 2013), ricas en recursos naturales, con un conjunto de características comunes que las ha convertido en casos típicos de comparación (Álvarez, 2007; Álvarez \& Bértola, 2013; Bertoni \&Willebald, 2016; Kirby, 1975; Schlüter, 2014). Entre sus principales similitudes destacan el tamaño de sus poblaciones y de sus mercados, la dotación de recursos naturales, la superficie destinada a la producción agraria, y el patrón de especialización productivo y de inserción en la economía mundial.

\section{GRÁFICO 1}

Relación PBI per cápita Uruguay/Nueva Zelanda, 1870-2010

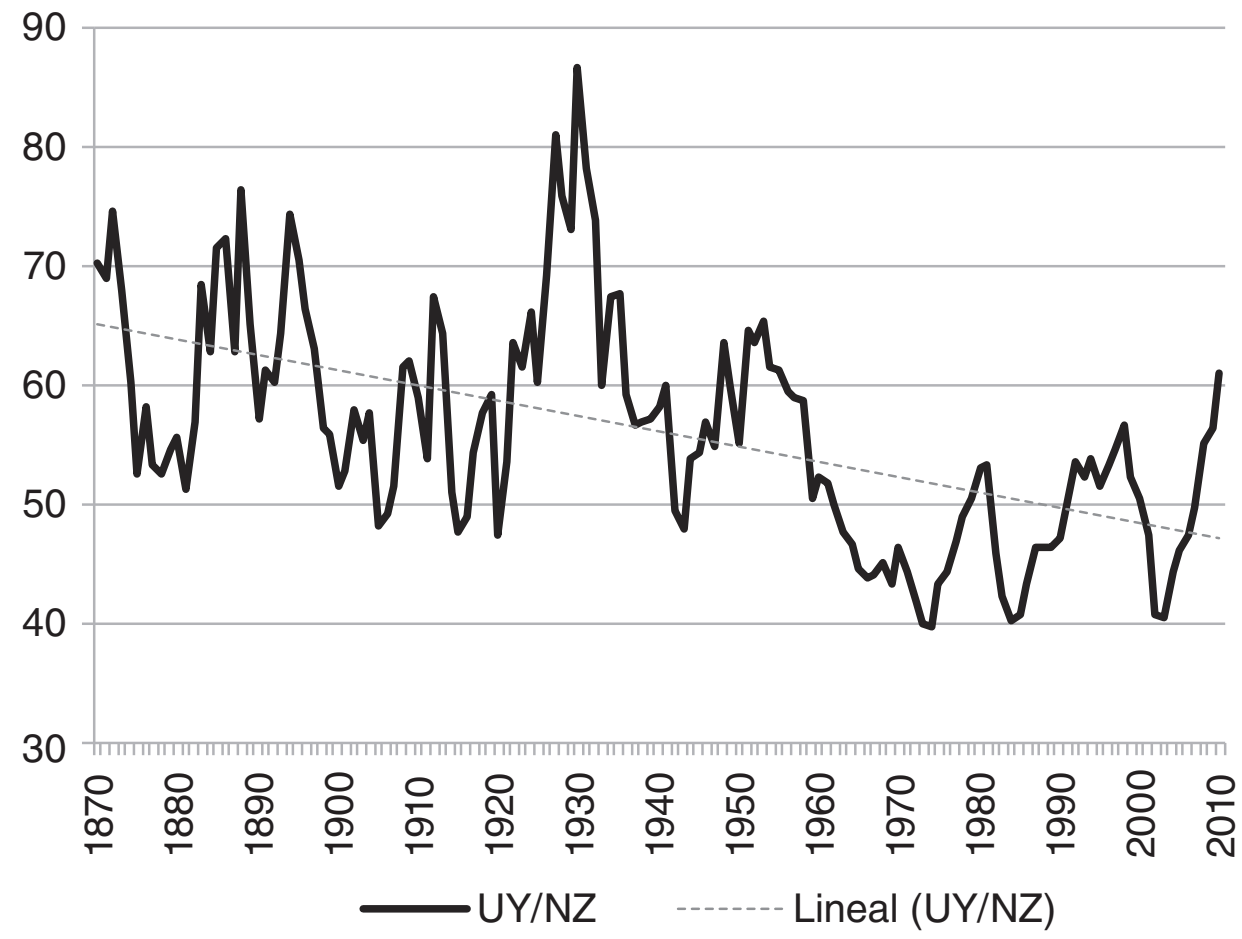

Fuente: con base en Maddison (2009) y Maddison-Project (2013).

Ambos países basaron su desarrollo a largo plazo en la configuración de una estructura productiva altamente especializada y en la exportación de un rango limitado de productos agrarios derivados de la actividad ganadera (cuero vacuno, lana, carne, lácteos), cuyos principales mercados fueron las economías desarrolladas de Europa (Bonino-Gayoso, 
Tena-Junguito \& Willebald, 2015b; Briggs, 2003; Finch, 2005). Este patrón de inserción en la economía mundial contribuyó a que ambos países alcanzaran altos niveles de ingreso por habitante hacia 1900 e implementaran innovaciones institucionales y reformas sociales avanzadas para la época (Bertram, 2009; Finch, 2005), debido a las oportunidades que la primera globalización del capitalismo brindó a las pequeñas economías agroexportadoras de clima templado con excelentes condiciones naturales para la producción agraria (Aparicio et al., 2018; Lewis, 1983).

No obstante estas similitudes, ambos países exhiben desempeños divergentes a largo plazo en variables clave de sus economías, como el PBI por habitante, la productividad agraria y el volumen de sus exportaciones.

En 1870 el PBI per cápita de Uruguay se ubicó en el entorno del 70\% del PBI per cápita de Nueva Zelanda; en la última década del siglo xx y primera del siglo XXI no supera, en promedio, el 50\%. El rezago de la economía uruguaya con relación a la neozelandesa se hizo más evidente a partir de la década de 1930 y se profundizó a partir de la década de 1950 (Gráfico 1).

\section{CUADRO 1}

Desempeño relativo de la productividad de los sectores agrarios de Nueva Zelanda y Uruguay, 1918-2010

\begin{tabular}{cccc}
\hline VAB agro & $\begin{array}{c}\text { VAB agro por trabajador } \\
\text { NZ/UY }\end{array}$ & $\begin{array}{c}\text { VAB agro por hectárea } \\
\text { NZ/UY }\end{array}$ \\
\hline 1918 & 1,11 & 1,24 & 1,07 \\
1930 & 1,16 & 1,39 & 1,10 \\
1970 & 2,10 & 2,85 & 2,00 \\
2010 & 1,58 & 1,27 & 1,79 \\
\hline
\end{tabular}

*A los efectos de la comparación, se considera la participación del sector agrario en el PBI de cada país expresado en dólares internacionales con base en Maddison Project (2013).

Fuentes: para el VAB del sector agrario en Nueva Zelanda, Bloomfield (1984), Edwards y Holmes (1994), Lineham (1968), Philpott y Hussey (1969), SNZ (2011); para el de Uruguay, Bertino y Tajam (1999), Bértola (2016), Bértola et al. (1998), Bonino, Román y Willebald (2012), BROU (1965); para los trabajadores en Nueva Zelanda, Bloomfield (1984), Briggs (2003), SNZ (2012a); para los trabajadores en Uruguay en 1908-1966, Bértola (2005) con base en Ardente, Díaz y Rossi (2004); 1970-2010, censos agropecuarios. En ambos países se considera al trabajador asalariado (a tiempo completo y ocasional), a los propietarios de los predios o a los que dirigen el establecimiento (gerentes o arrendatarios). Se excluye el trabajo familiar no remunerado. Para la superficie destinada a la producción agraria en Nueva Zelanda, SNZ (2012a), varios años; en Uruguay, censos agropecuarios, varios años (1918 corresponde a la superficie reportada en el censo de 1916).

Un factor clave del desempeño divergente de ambas economías ha sido el crecimiento de la productividad agraria. Hacia fines de la Primera Guerra Mundial, Nueva Zelanda ape- 
nas aventajaba a Uruguay en los niveles del PBI agrario, así como en la productividad por trabajador y por hectárea (Cuadro 1). Sin embargo, a comienzos de la década de 1970, Nueva Zelanda duplicó el valor agregado bruto (VAB) del sector agrario y elVAB por hectárea de Uruguay, y triplicó elVAB del agro por trabajador. Posteriormente, en el último cuarto del siglo $\mathrm{xx}$, Uruguay mejoró el desempeño relativo de la productividad del sector, sin por ello alcanzar los niveles de Nueva Zelanda.

\section{GRÁFICO 2}

Participación de productos ganaderos en las exportaciones totales de Nueva Zelanda y Uruguay, 1870-2010 (\%)

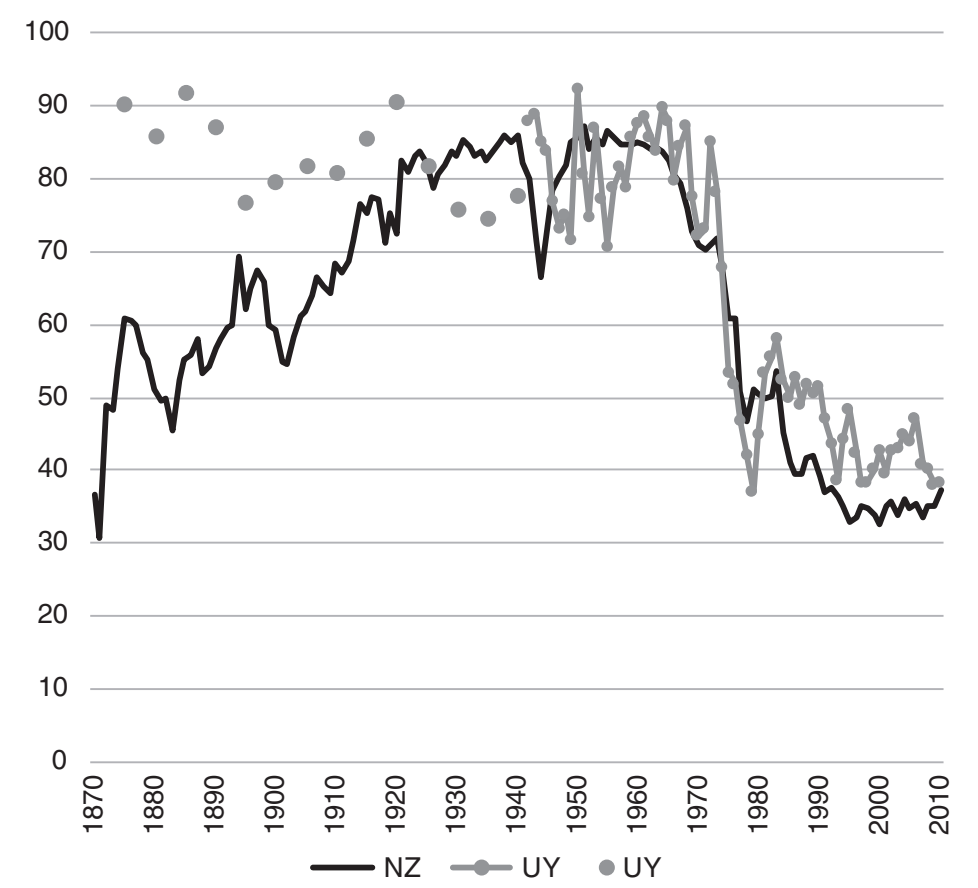

Fuentes: Nueva Zelanda, 1870-2006, Briggs (2003); 2007-2010, SNZ (2012a). Uruguay, 1892-1941, promedios quinquenales con base en 1872-1890, Millot y Bertino (1996: cuadro IV.3, p. 138); 1891-1941, Finch (2005: cuadro 4.4, p. 162); serie anual con base en 1942-1968, Instituto de Economía (1969: cuadro 39); 1969-1971, BCU (1973: n. ${ }^{\circ} 12$, cuadro 15, p. 40); 1972-1974, BCU (1979: n. ${ }^{\circ} 25$, cuadro 11, p. 26); 19751992, boletines estadísticos, años respectivos tomado de FCS (2013); 1993-2010 Banco Central del Uruguay, series tomadas de INE (2013).

Si se tiene en cuenta que la ganadería ha sido el principal subsector del agro en ambos países ${ }^{1}$, que más del $80 \%$ de las exportaciones uruguayas y más del $70 \%$ de las ex-

1. La ganadería representa, en promedio, más del $70 \%$ del PBI agrario de Uruguay entre 1870 y 2010 (BERTINO \& TAJAM, 1999; BÉRTOLA et al., 1998; Sistema de Cuentas Nacionales). La informa- 
portaciones neozelandesas entre 1870 y 1970 fueron, en promedio, productos derivados de la ganadería (Gráfico 2) y que Uruguay verificó un estancamiento de sus exportaciones entre las décadas de 1930 y 1970, en tanto Nueva Zelanda mantuvo una tendencia creciente a largo plazo (Gráfico 3), el análisis del desempeño productivo del sector ganadero se torna crucial para comprender el desempeño a largo plazo de ambas economías.

El principal objetivo del artículo -que forma parte de un proyecto de investigación más amplio $^{2}$ - es identificar las principales tendencias históricas del desempeño relativo de la producción ganadera de ambos países, ampliando la base de información cuantitativa sobre productividad física que ofrecen anteriores trabajos comparativos ${ }^{3}$. También brindar una explicación de estas tendencias considerando los procesos de incorporación de tecnologías mejoradoras del factor tierra en cada país.

Los antecedentes pusieron de relieve dos grandes problemas al comparar el desempeño de la ganadería de Nueva Zelanda y Uruguay: en primer lugar, la comparabilidad de los indicadores sintéticos de productividad física de uso común en economía agraria y en historia agraria, como la unidad ganadera (UG) y la carne equivalente (CE); y, en segundo lugar, la necesidad de incorporar al análisis indicadores de productividad de la tierra dedicada a la producción ganadera en las escalas nacional y regional, dadas las diferencias de aptitud de la tierra para la producción ganadera que exhiben las distintas regiones de cada país. En este artículo se busca atender ambos problemas a través de una comparación exhaustiva de los distintos rubros de producción ganadera (stock animal, composición del stock, producción de carne, lana y leche, superficie de pastoreo y recursos forrajeros) y de la revisión de los coeficientes técnicos aplicados a los indicadores sintéticos de productividad física. También esbozar algunas hipótesis sobre el dispar desempeño de la ganadería en ambos países con base en los conceptos de trayectoria y paradigma tecnológico, desarrollados por los enfoques evolucionistas del cambio técnico.

ción disponible para Nueva Zelanda indica que la ganadería representa más del $60 \%$ del PBI agrario entre 1971 y 2010, y más del 80\% del valor bruto de producción del sector entre 1921 y 1967 (Philpott \& Hussey, 1969).

2. Vease Álvarez (2014)

3. Especialmente la información de largo plazo que brindan Álvarez y BorTAGARAY (2007) y ÁLVAREZ (2008). DAVIE (1960) y GALLINAL (1951) destacaron las diferencias técnicas de la producción ganadera de ambos países en la década de 1950. KIRBY (1975) enfatizó el papel de la estructura de la propiedad de la tierra en el desempeño del sector agrario. 


\section{GRÁFICO 3}

Índice del volumen de las exportaciones

de Nueva Zelanda y Uruguay, 1870-2010 (log $1913=100)$

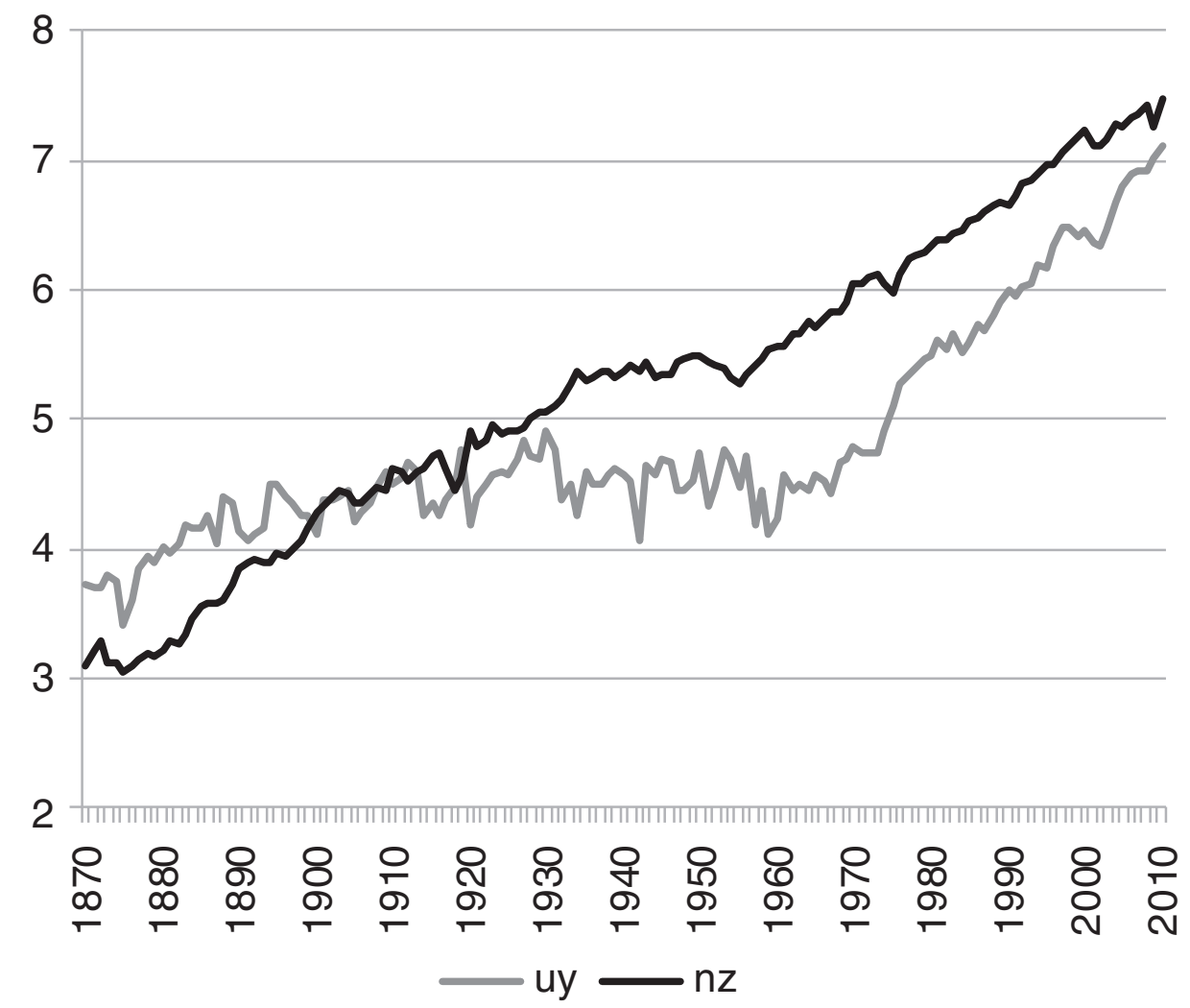

Fuentes: Nueva Zelanda, 1870-2005, exportaciones reales Briggs (2003); 2006-2010, índice de volumen de exportaciones, World Bank (2014). Uruguay, 1870-1913, Bonino-Gayoso, Tena-Junguito y Willebald (2015a; 2015b: 71, tabla F1); 1913-1970, Finch (2005: 159-161, cuadros 4.1, 4.2, 4.3); 1970-2010, World Bank (2014).

Luego de esta introducción, en la Sección 2 se presenta información sobre el stock animal y el uso del suelo en Nueva Zelanda y Uruguay. En la Sección 3, se comparan las principales tendencias de la productividad física de la ganadería, en las escalas nacional y regional, a través de indicadores sintéticos de productividad física como la UG y la CE, para lo cual se proponen una serie de correcciones a los factores de ponderación comúnmente utilizados para la agregación de los distintos rubros de la producción ganadera. En la Sección 4 se presenta una hipótesis sobre el desempeño divergente de la ganadería en ambos países y se identifican las principales trayectorias tecnológicas de la ganadería asociadas al desarrollo de tecnologías mejoradoras del factor tierra. Finalmente, se presentan las 
principales conclusiones en la Sección 5. En un Anexo ${ }^{4}$ se detalla la información estadística que sirve de base para la comparación.

\section{PRINCIPALES TENDENCIAS: ÁREA DE PASTOREO Y STOCK ANIMAL EN NUEVA ZELANDA Y URUGUAY}

Los sistemas ganaderos de Nueva Zelanda y Uruguay tienen tres grandes similitudes: la ubicación de ambos países en las regiones templadas del hemisferio sur ${ }^{5}$, lo que les ha permitido gozar de un clima apto para la producción ganadera a cielo abierto durante todo el año; el pastoreo en pradera como base de la alimentación animal; y la superficie territorial dedicada al pastoreo animal. También un conjunto de diferencias geográficas, como la relación área productiva-territorio (90\% en Uruguay, 50\% en Nueva Zelanda), la topografia (llanuras y penillanuras en Uruguay, regiones montañosas en Nueva Zelanda) y los cambios experimentados por el ecosistema nativo (véase Sección 4).

Como muestran los Gráficos 4 y 5, la superficie de pastoreo en ambos países fue similar a partir de la primera década del siglo xx. En Nueva Zelanda hubo un fuerte crecimiento del área dedicada a la ganadería entre 1870 y 1911, que pasó de 6,3 a 12,6 millones de hectáreas como consecuencia de la incorporación de vastas regiones deforestadas y convertidas en praderas en la North Island. Posteriormente, el área de pastoreo permaneció en similares niveles hasta alcanzar el pico máximo de 14 millones de hectáreas en 1980. En Uruguay la superficie de pastoreo ha sido relativamente estable, oscilando en el entorno de los 13-15 millones de hectáreas a lo largo del siglo xx. Desde el punto de vista de la composición del stock, la relación entre el stock ovino y el bovino fue tradicionalmente más alta en Nueva Zelanda que en Uruguay.

El Cuadro 2 compara el stock animal y el stock de tierra dedicado a la ganadería en años elegidos ${ }^{6}$ y las tasas de crecimiento acumulativo anual por períodos. En él se observa que Nueva Zelanda tuvo tasas de crecimiento del stock animal y de la superficie de pastoreo más altas que Uruguay, especialmente en el período 1870-1970. También que el crecimiento del stock animal en Nueva Zelanda, tanto bovino como ovino, fue mayor que el crecimiento de la superficie dedicada al pastoreo, lo que sugiere un importante creci-

4. El anexo está publicado en la versión online en la página web de la revista DOI https://doi.org/10.26882/histagrar.080x06a

5. Latitud norte-sur: $\mathrm{NZ}, 35^{\circ}-47^{\circ}, \mathrm{UY}, 30^{\circ}-35^{\circ}$.

6. Los años elegidos corresponden a puntos de inflexión de los principales modelos de crecimiento de cada país. 
miento de la productividad de la tierra. En Uruguay el crecimiento del stock animal y de la tierra muestra valores próximos a $0 \%$ hasta 1930; posteriormente un leve crecimiento del stock bovino y una caída del stock ovino.

\section{GRÁFICO 4}

Nueva Zelanda: evolución del stock ganadero y superficie de pastoreo, 1870-2010

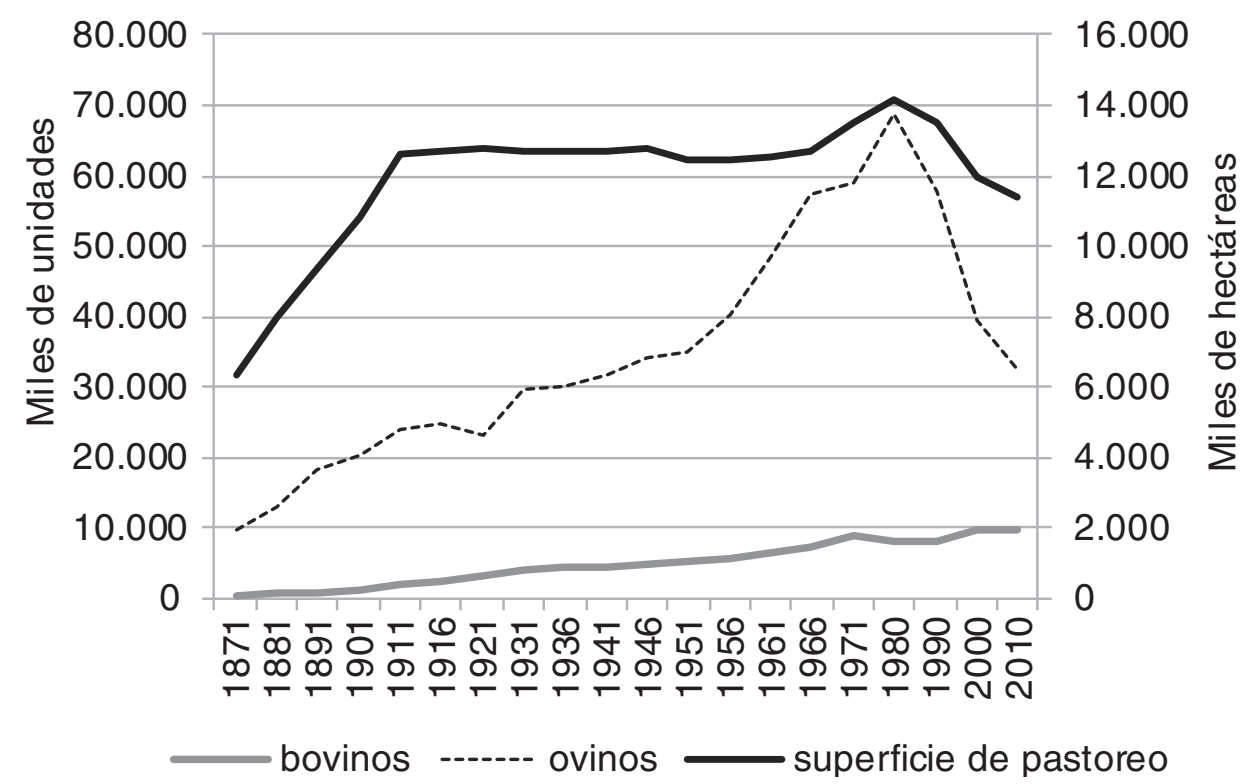

Nota: eje izquierdo, bovinos y ovinos en miles de unidades; superficie de pastoreo en miles de hectáreas en el eje derecho.

Fuentes: Cuadro A1 del Anexo.

Más allá de la información que brinda el Cuadro 2, para comparar el crecimiento de la productividad física de la tierra considerando ambas especies ganaderas, es preciso traducir los respectivos stocks a un indicador sintético como la unidad ganadera (UG). Por otra parte, para comparar la productividad física de los principales rubros de la producción ganadera (lana, carne, leche), tanto con referencia a la tierra como a la propia UG, es preciso traducir cada rubro de producción a un producto de referencia, para el caso, la carne. En la próxima sección discutimos los criterios de agregación aplicados en cada sistema agrario en términos de UG y de carne equivalente (CE) a lo largo del tiempo y proponemos nuevas series históricas para cada país. 


\section{GRÁFICO 5}

Uruguay: evolución del stock ganadero y superficie de pastoreo, 1870-2010

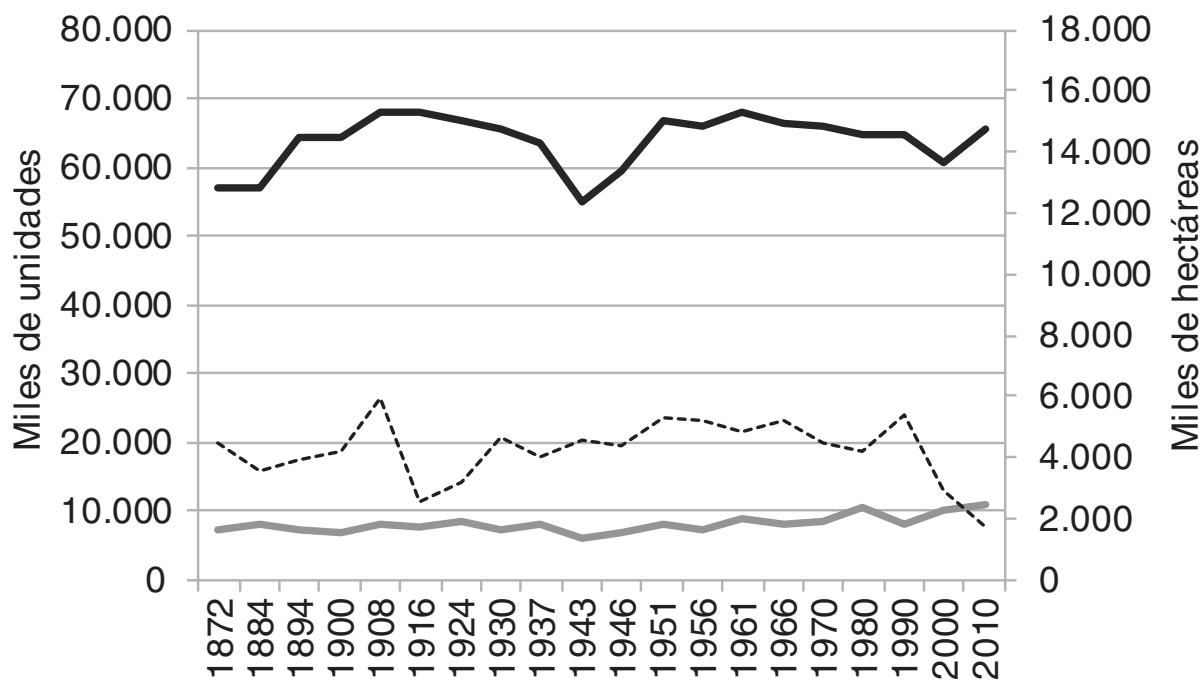

bovinos ------ ovinos $\longrightarrow$ superficie pastoreo

Nota: eje izquierdo, bovinos y ovinos en miles de unidades; superficie de pastoreo en miles de hectáreas en el eje derecho.

Fuentes: Cuadro A1 del Anexo.

CUADRO 2

Stock animal y tierra (millones) y tasas de crecimiento (\%)

\begin{tabular}{lccccccc}
\hline & \multicolumn{3}{c}{ Nueva Zelanda } & \multicolumn{3}{c}{ Uruguay } \\
\hline & $\begin{array}{c}\text { Bovino } \\
\text { cabezas }\end{array}$ & $\begin{array}{c}\text { Ovino } \\
\text { cabezas }\end{array}$ & $\begin{array}{c}\text { Tierra } \\
\text { hectáreas }\end{array}$ & & $\begin{array}{c}\text { Bovino } \\
\text { cabezas }\end{array}$ & $\begin{array}{c}\text { Ovino } \\
\text { cabezas }\end{array}$ & $\begin{array}{c}\text { Tierra } \\
\text { hectáreas }\end{array}$ \\
\hline 1871 & 0,4 & 9,7 & 6,4 & 1872 & 7,2 & 20,0 & 12,8 \\
1931 & 4,1 & 29,8 & 12,7 & 1930 & 7,1 & 20,6 & 14,8 \\
1971 & 8,8 & 58,9 & 13,5 & 1970 & 8,6 & 19,9 & 14,8 \\
2010 & 9,9 & 32,6 & 11,4 & 2010 & 11,1 & 7,7 & 14,8 \\
\hline \multicolumn{1}{l}{} & \multicolumn{1}{c}{ Tasas de crecimiento (\%) } & & Tasas de crecimiento (\%) \\
\hline $1871-1931$ & 3,8 & 1,9 & 1,2 & $1872-1930$ & 0,0 & 0,0 & 0,3 \\
$1931-1970$ & 1,9 & 1,7 & 0,2 & $1930-1970$ & 0,5 & $-0,1$ & 0,0 \\
$1970-2010$ & 0,3 & $-1,5$ & $-0,4$ & $1970-2010$ & 0,6 & $-2,3$ & 0,0 \\
\hline
\end{tabular}

Fuente: elaboración propia con base en las fuentes de los Gráficos 4 y 5 . 


\section{PRINCIPALES TENDENCIAS DE LA PRODUCTIVIDAD FÍSICA DE LA GANADERÍA}

\subsection{Unidad ganadera: coeficientes de equivalencia y aplicación en los sistemas ganaderos de Nueva Zelanda y Uruguay}

La UG es un coeficiente técnico de uso común en estadística y economía agraria con el que se realizan análisis globales y comparativos de explotaciones ganaderas. La idea básica es determinar la carga animal que puede soportar un campo en los meses de invierno ${ }^{7}$ en función de los requerimientos energéticos y las necesidades alimentarias del ganado, considerando que en ambos países se produce el pastoreo conjunto de ovinos y bovinos. El cálculo de UG toma como referencia una especie animal ${ }^{8}$, multiplicando el número de cabezas reales de ganado por un factor de ponderación. Este factor se estima con base en la cantidad anual de alimento que requiere cada especie considerando su peso vivo (Aguirrezabala, 1989; Woodford \& Nicol, 2004).

En Nueva Zelanda el concepto UG fue usado por primera vez por Fawcett y Patton (1929) en un artículo publicado en CSO (1929) en el que se evaluó el desempeño agrario de ese país en las primeras décadas del siglo xx. La relación de equivalencia usada en esa ocasión fue seis ovinos por bovino productor de carne (CSO, 1929: tabla 6). Sin embargo, el uso del concepto livestock unit se extenderá en Nueva Zelanda recién a partir de la década de 1970. El trabajo que dio gran impulso al uso del indicador fue realizado por Coop (1965), quién reportó un coeficiente de equivalencia ovino/bovino 6:1. El sistema de equivalencia con base en los trabajos de Coop fue revisado en diversos trabajos (Cornforth \& Sinclair, 1984; Fleming, 2003; Parker, 1998; Woodford \& Nicol, 2004), y destaca la revisión realizada por el MAF (Ministry of Agriculture and Forestry) en 1992 (Cuadro 3).

\section{CUADRO 3}

Nueva Zelanda: coeficientes de equivalencia ganadera

\begin{tabular}{lcrrrrrr}
\hline & Fawcett \& Patton & Curry & \multicolumn{1}{c}{ Coop } & Tennant & \multicolumn{2}{c}{ MAF Fleming } & Woodford \& Nicol \\
\hline Bovino (carne) & 1 & 1 & 1 & 1 & 1 & 1 & 1 \\
Bovino (leche) & 1 & 0,85 & $0,9-0,7$ & 0,75 & 1 & 0,65 & 1 \\
Ovino & 6 & 5,5 & 6 & 6 & 5,5 & 4,5 & 6,6 \\
\hline
\end{tabular}

Fuentes: Fawcett y Paton (1929); Curry (1963); Coop (1965); Tennant (1978); MAF (1992) citado en INIA (2012); Fleming (2003); Woodford y Nicol (2004).

7. En los casos de Nueva Zelanda y Uruguay los cálculos de la carga animal se realizan al 30 de junio de cada año (DALTON, 2009; INIA, 2012).

8. Es una convención en Uruguay tomar como especie de referencia al ganado bovino adulto de cría. En Nueva Zelanda el animal de referencia es el ovino adulto de cría. 


\section{CUADRO 4}

Uruguay: coeficientes de equivalencia ganadera

\begin{tabular}{lcccccr}
\hline & CLAEH-CINAM OPYPA-CIDE & MGA & Coneat $^{9}$ & Aguirrezabala\&Oficialdegui & Boné\&Perugorría & INIA \\
\hline Bovino (carne) & 1 & 1 & 1 & 1 & 1 & 1 \\
Bovino (leche) & 1 & 1 & 1 & 1 & 1 & 1 \\
Ovino & 5 & 5 & 6 & 6,34 & 6 & 6,67 \\
\hline
\end{tabular}

Fuentes: CLAEH-CINAM (1963); OPYPA-CIDE (1967); MGA (1973) citado en Crempien (1982); CONEAT (1979); Aguirrezabala y Oficialdegui (1995); Boné y Perugorría (2011); INIA (2012).

En Uruguay el coeficiente de ponderación más utilizado entre ovinos y bovinos ha sido 5:1. Esta relación fue ampliamente usada para estimar la carga animal del área total de pastoreo del país y para el análisis de gestión en el nivel de los establecimientos. También ha sido aplicada en diversos trabajos que han realizado análisis globales, históricos y de largo plazo del sector ganadero uruguayo sin referir ni discutir la base científica que lo sustenta (CLAEH-CINAM, 1963: 60, cuadro A-III.-21; OPYPA-CIDE, 1967: 351, cuadro 27). La acumulación de evidencia científica que cuestiona el coeficiente de equivalencia clásico 5:1 en Uruguay impulsó un amplio estudio (INIA, 2012), que involucró a diversas organizaciones públicas y privadas comprometidas con el sector agrario ${ }^{10}$. Los principales resultados indican que los requerimientos nutricionales de los ovinos han sido sobrevalorados, por lo que se propone utilizar una nueva relación ovino/bovino de 6,67:1, siendo una oveja adulta de cría 0,15 UG (INIA, 2012).

El Cuadro 4 sintetiza los coeficientes de equivalencia ovino/bovino aplicados en Uruguay y reportados en distintos trabajos.

La decisión de aplicar distintos coeficientes de equivalencia a lo largo del tiempo no es trivial, porque el resultado afecta el nivel histórico de las UG de cada país, especialmente si se compara con los resultados que muestran anteriores trabajos. En el caso de Uruguay, el cambio propuesto supone, a partir de la década de 1970, un cambio a la baja del nivel de las UG por hectárea reportadas por Astori (1979), Moraes (2001, 2008), y

9. La Comisión Nacional de Estudio Agronómico de la Tierra (CONEAT) fue creada en 1968 con el objetivo de fijar, mediante un índice, la capacidad productiva media del país y de los predios rurales en el marco de la implementación de un nuevo régimen impositivo capaz de gravar la productividad intrínseca de la tierra y no su producción. La Comisión estableció un coeficiente de equivalencia ovino-bovino y estimó la productividad de cada padrón rural en términos de lana y carne (bovina y ovina) (LANFRANCO \& SAPRIZA, 2011).

10. Ministerio de Ganadería Agricultura y Pesca, Facultades de Agronomía y Veterinaria de la Universidad de la República, Federación Uruguaya de los Grupos Crea, Instituto Nacional de Investigación Agropecuaria, Instituto Plan Agropecuario, Secretariado Uruguayo de la Lana. 
Álvarez y Bortagaray (2007). En el caso de Nueva Zelanda, supone un cambio de nivel también a la baja según lo reportado por Álvarez y Bortagaray (2007) y Brooking y Pawson (2011), en particular, en el período 1870-1961. Como se verá, si bien estos cambios no alteran las grandes tendencias, capturan de forma más precisa las transformaciones experimentadas por el sector, especialmente desde la década de 1960 en adelante cuando comenzaron a reportarse revisiones de los coeficientes de equivalencia ovino/bovino para el cálculo de la UG.

\section{CUADRO 5}

Unidad ganadera: coeficientes de equivalencia utilizados, fuentes y períodos

\begin{tabular}{|c|c|c|c|c|c|}
\hline NuevaZelanda & Fawcett \& Patton (1929) & Coop (1965) & Tennant (1978) & MAF (1992) Woo & ford \& Nicol (2004) \\
\hline & $1871-1956$ & $1961-1970$ & 1980 & $1990-2000$ & 2010 \\
\hline \multirow[t]{2}{*}{ Uruguay } & CLAEH-CINAM (1963) & CONEAT (1979) & \multicolumn{2}{|c|}{ Aguirrezabala \& Oficialdegui (1995) } & INIA (2012) \\
\hline & $1872-1966$ & $1970-1980$ & \multicolumn{2}{|c|}{$1990-2000$} & 2010 \\
\hline
\end{tabular}

Nota: cada período inicia con el primer coeficiente de equivalencia reportado. Si el período anterior es muy extenso o si la publicación del nuevo coeficiente sucede en pocos años al último censo, se aplica el nuevo coeficiente al censo inmediatamente anterior. La existencia de distintos coeficientes de equivalencia en cada sistema ganadero a lo largo del tiempo, pone de manifiesto la inconveniencia de utilizar un coeficiente fijo en el largo plazo, como ha sido de uso corriente en ambos países (Álvarez \& Bortagaray, 2007; Astori, 1979; Brooking \& Pawson, 2011; Moraes, 2008). Especialmente si se tiene en cuenta que el sector agrario, y en particular la ganadería, experimentó grandes transformaciones a lo largo del período, entre las que se destacan, cambios en la composición genéticas de los animales, la introducción de nuevas razas y las consecuentes variaciones de los requerimientos energéticos de las distintas especies. Para enfrentar este problema proponemos aplicar distintos coeficientes de equivalencia a lo largo del tiempo en cada país (Cuadro 5), asumiendo que los coeficientes de equivalencia reportados en los Cuadros 3 y 4 reflejan de mejor forma la relación ovino/bovino del período en el que fueron propuestos.

Fuente: elaboración propia.

\subsection{Productividad física de la tierra de uso ganadero en términos de unidades ganaderas}

El Gráfico 6 muestra la evolución de las unidades ganaderas por hectárea (UG/ha) y la evolución de la relación UG/ha de Nueva Zelanda y Uruguay aplicando, por un lado, las correcciones propuestas al indicador y, por el otro, coeficientes de equivalencia fijos (relación ovino/bovino 5:1). Si bien las tendencias del desempeño relativo de las UG/ha con coeficientes fijos y con los nuevos coeficientes propuestos son, grosso modo, coincidentes (véanse variables NZ/UY y NZ/UY coeficiente fijo en el Gráfico 6), en la nueva estimación se verifica una caída del nivel de la relación UG/ha hasta la década de 1950 y posteriormente fluctuaciones en torno a similares niveles. 


\section{GRÁFICO 6}

\section{Evolución de las unidades ganaderas por hectárea}

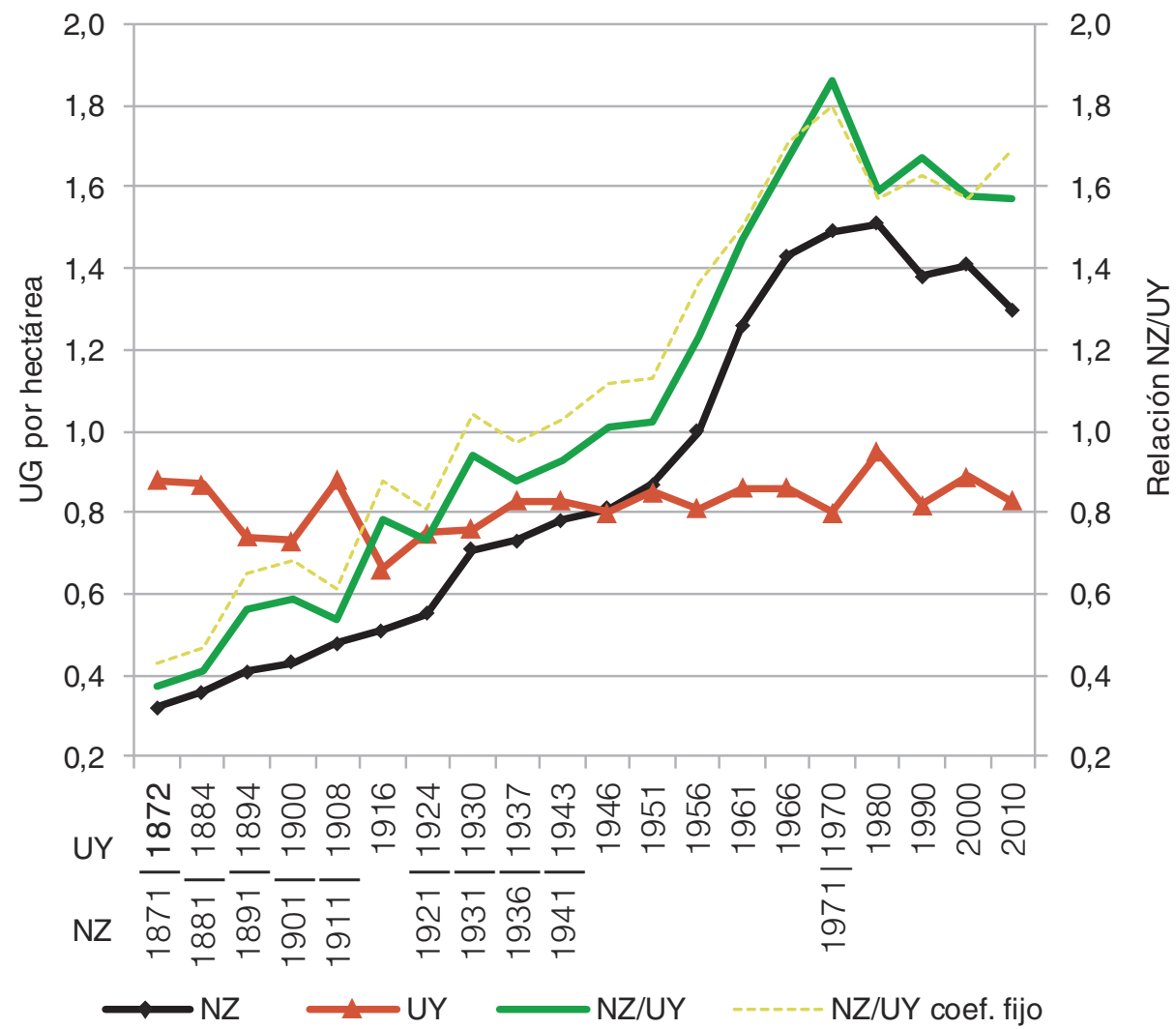

Nota: coeficientes de equivalencia aplicados en Nueva Zelanda y Uruguay según se detalla en el Cuadro 5. Los ratios NZ/UY fueron calculados tomando la información surgida de los censos y de los anuarios estadísticos de cada país con base en las fuentes reportadas en el Anexo. Cuando los años no coinciden, la relación se calcula considerando los años censales más próximos de cada país. En el eje horizontal se muestra el año correspondiente al dato de cada país.

Fuentes: todas las fuentes de los cuadros están reportadas en el Anexo. Para Nueva Zelanda, área de pastoreo, Cuadro A1; stock animal, Cuadro A2. Para Uruguay, área de pastoreo y stock animal, Cuadro A4; coeficientes de equivalencia, cuadros 3 y 4.

Las principales tendencias indican que Nueva Zelanda experimentó un crecimiento constante de las UG/ha desde 1871 hasta 1980, con dos períodos de fuerte crecimiento (década de 1920, y entre 1951 y 1966) y una tasa de crecimiento a largo plazo (18702010) cercana al 1\% acumulativo anual. En el caso de Uruguay, la tendencia predominante ha sido el estancamiento en torno a los mismos niveles, más allá de las fluctuaciones registradas entre 1871 y 1930 y del leve crecimiento posterior a la década de 1970, con tasas de crecimiento a largo plazo de las UG/ha próximas al 0\%. 
MAPA 1

Regiones consideradas en Nueva Zelanda y Uruguay

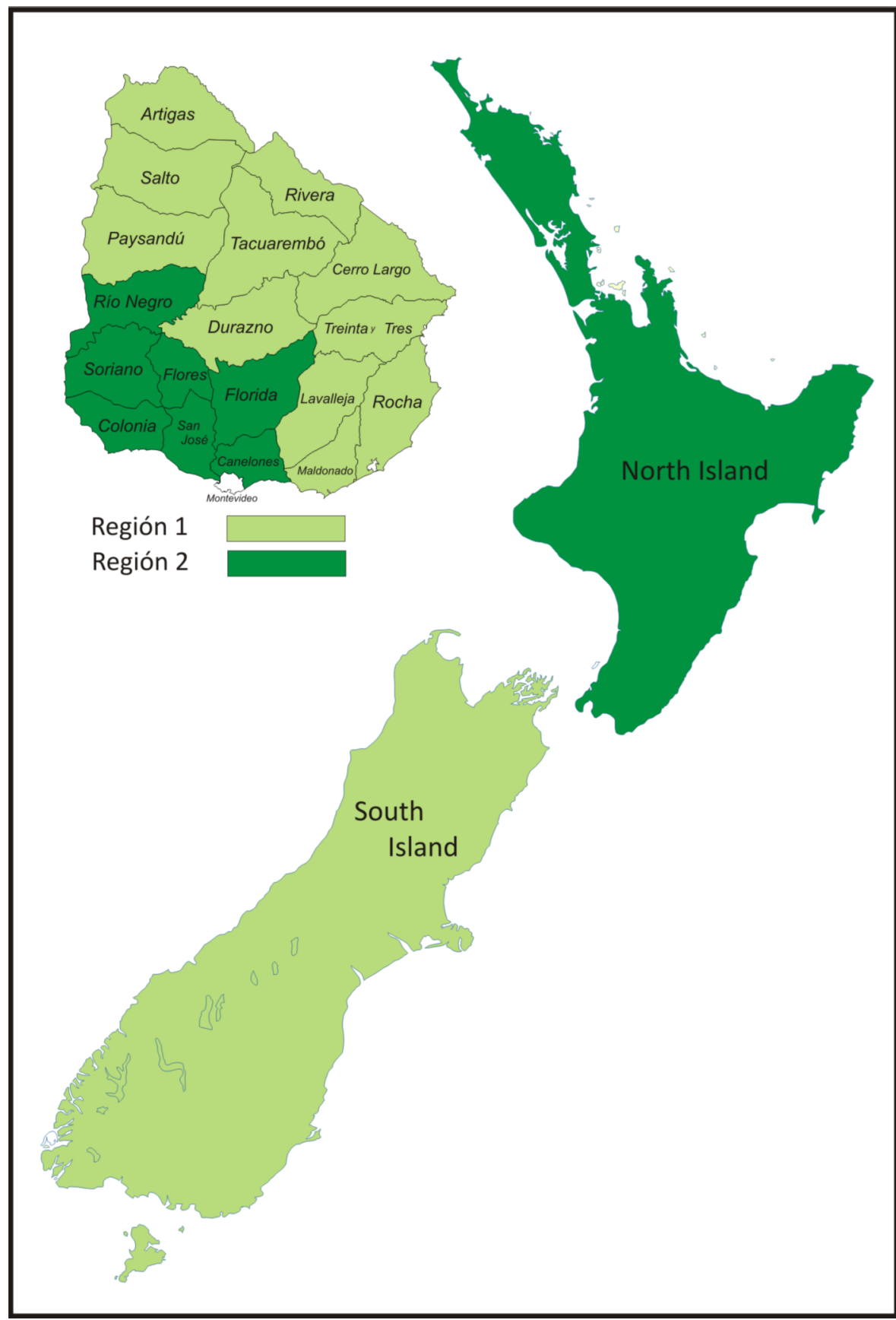

Fuente: elaboración propia. 
No obstante estas tendencias, Uruguay tuvo una carga animal por hectárea más alta que Nueva Zelanda hasta la década de 1930. Entre 1871 y 1911, Uruguay duplicó en promedio las UG/ha de Nueva Zelanda. Posteriormente la brecha se acortó hasta que en la década de 1940 Nueva Zelanda alcanzó los niveles de Uruguay. Entre 1951 y 1971 el fuerte crecimiento de las UG/ha en Nueva Zelanda le permitió casi duplicar los niveles de Uruguay. A partir de 1980 la brecha se redujo, aunque Nueva Zelanda conservó hasta el fin del período niveles un $60 \%$ superiores, en promedio, a los de Uruguay.

\subsection{Productividad física de la tierra de uso ganadero a escala regional}

Los niveles, las tendencias y el desempeño relativo de las UG/ha a escala nacional no coinciden completamente cuando se introduce en el análisis comparado la escala regional. De allí que resulta relevante comparar la productividad parcial de la ganadería en términos de UG/ha entre regiones. En el caso de Nueva Zelanda, el enfoque regional es insoslayable debido a las diferencias que presentan la North Island y la South Island, en términos de los respectivos procesos de expansión agraria y de sus características agroecológicas (Taylor \& Smith, 1997). En el caso de Uruguay, el enfoque regional también es relevante debido a las diferencias de aptitud de los suelos y de especialización por rubro principal (agrícola o ganadero) de las dos principales regiones del país, suroeste y noreste (DIEA, 2015). El Mapa 1 detalla las regiones de cada país que sirven de base para la comparación, y el Gráfico 7 presenta la productividad de la tierra en términos de UG a escala regional.

En el caso de Nueva Zelanda, lo primero que se observa son marcadas diferencias a escala regional. El nivel de UG/ha en la South Island fue bajo entre las décadas de 1870 y 1940, en promedio 0,36 UG/ha, y creció entre las décadas de 1950 y 1980 de 0,43 a $0,9 \mathrm{UG} / \mathrm{ha}$. En la North Island hubo un crecimiento constante de la productividad física de la tierra desde 1871 a 1966 . Entre 1871 y 1911 el proceso combinó crecimiento extensivo del área de pastoreo (de 1,8 a 6 millones de hectáreas) con aumento de la productividad de la tierra en términos de UG/ha. En el período 1921-1966 hubo un sostenido aumento de la UG/ha: en 1921 la North Island podía sostener, en promedio, una carga de 1 UG/ha y en 1966 alcanzó el nivel histórico más alto, con 2,3 UG/ha.

En el caso de Uruguay las diferencias regionales no son tan marcadas como en Nueva Zelanda. Atendiendo a las características agronómicas de las distintas regiones del país para la producción ganadera, la Comisión Nacional de Estudio Agronómico de la Tierra (CONEAT, 1979) fijó la capacidad productiva media del país, y de cada región, a través de la construcción de un índice de productividad de la tierra (véase Nota 9). Este 
índice refleja la existencia de dos regiones: litoral suroeste y región noreste del territorio. Considerando la distribución regional que surge del índice CONEAT (1979), agrupamos los departamentos de Uruguay (las unidades jurisdiccionales del país consideradas en los censos agropecuarios) en dos grandes regiones que denominamos región 1 y región 2. La región 1 está conformada por los departamentos que presentan una productividad inferior al promedio nacional. La región 2 está conformada por los departamentos cuyo índice de productividad supera el promedio del país. El conjunto de los departamentos con productividad superior a la media nacional (región 2) presenta, en promedio, una carga animal por hectárea $20 \%$ superior a la de la región 1 hasta la década de 1960 , y un $10 \%$, en promedio, entre 1970 y 2010 . Si bien la diferencia entre las regiones no es menor, no es tan grande como la que exhiben la North Island y la South Island en Nueva Zelanda.
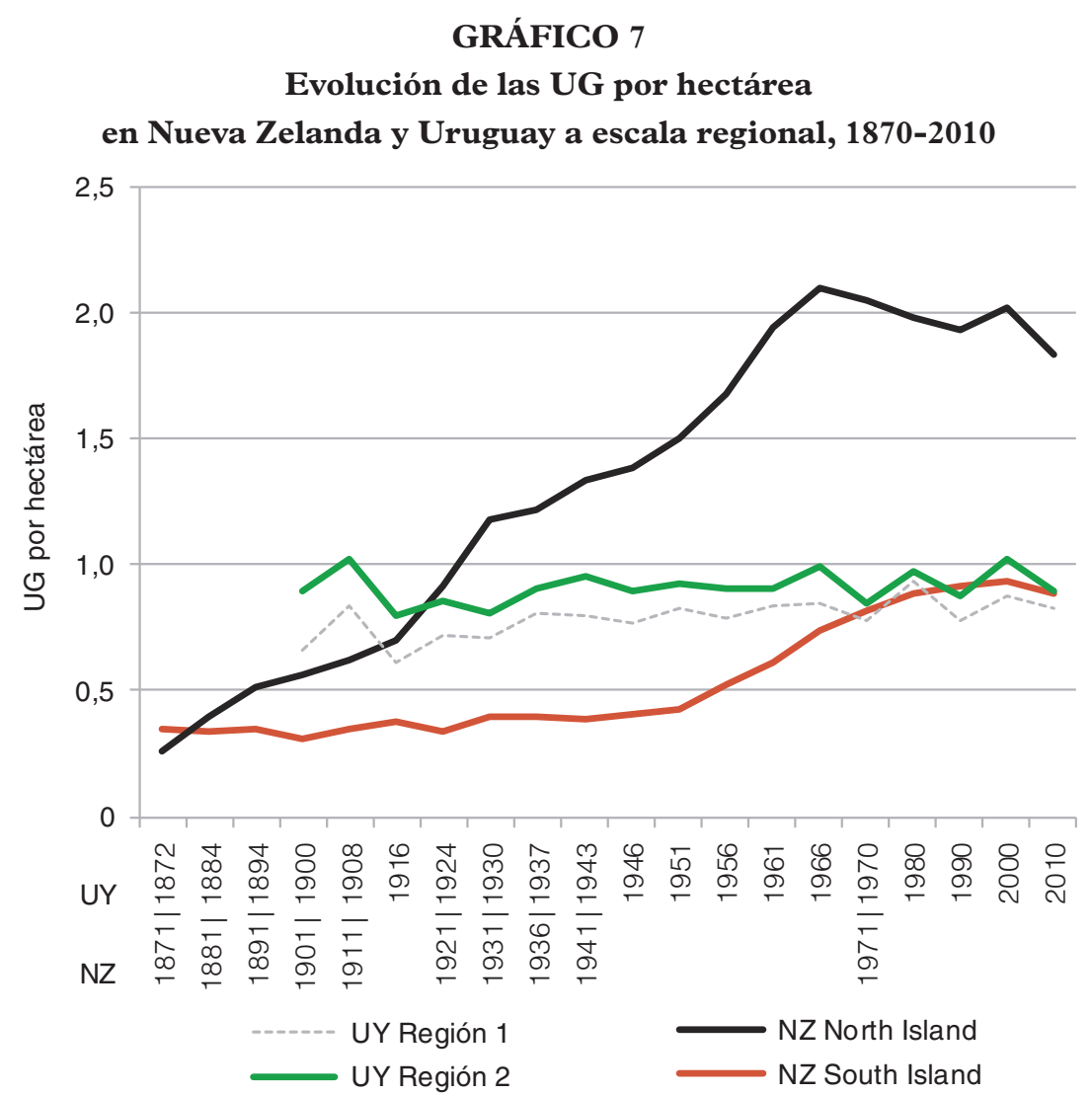

Fuente: coeficientes de equivalencia ovino/bovino según se detalla en Cuadro 5. Nueva Zelanda, área de pastoreo por isla, Cuadro A1 del Anexo; stock animal por isla, Cuadro A3 del Anexo. Uruguay, Cuadro A5 del Anexo. 
Lo primero que llama la atención, al comparar la productividad de la tierra de ambos países, es que el nivel de productividad de la tierra en la South Island es muy inferior al que presenta Uruguay. Hasta la década de 1950, Uruguay duplicó la carga animal por hectárea de la South Island. Este es un dato significativo si tenemos en cuenta que, en el largo plazo, cerca del $60 \%$ del área total de pastoreo de Nueva Zelanda estuvo localizada en la South Island. Entre 1950 y 1970, la South Island mejoró la productividad de la tierra ganadera y alcanzó los niveles de Uruguay en el último cuarto del siglo xx (véase Sección $4)$.

En segundo término, las mejoras verificadas en la North Island desde 1870 produjeron una convergencia más temprana con la ganadería uruguaya. La North Island exhibe más altos niveles de UG/ha que la región 2 de Uruguay desde la década de 1920 e incrementa esta diferencia hasta 1966, cuando duplica los niveles de Uruguay. Estas diferencias entre la North Island y la región 2 de Uruguay están relacionadas con los cambios tecnológicos vinculados a la producción de pasturas (véase Sección 4). El uso de la tierra en la North Island tuvo una clara especialización ganadera (la producción agrícola se orientó completamente a la producción de pasturas y forraje, véase Sección 4.1.2), en tanto la región 2 de Uruguay tuvo una especialización agrícola-ganadera ${ }^{11}$.

Sobre estas diferencias en las escalas nacional y regional volveremos en las secciones 4 y 5 .

\subsection{Productividad física de la tierra y de las unidades ganaderas en términos de carne equivalente}

Otro indicador sintético de productividad física de la ganadería es el concepto de carne equivalente (CE). Se trata de un indicador homogéneo de productividad física de la ganadería de uso corriente en Uruguay que resume la producción de los principales rubros

11. Entre 1901 y 2010, el 43\% (desvío estandar 2,5) de la superficie forrajera de Nueva Zelanda (campo natural, praderas artificiales y cultivos forrajeros) se localizó en la North Island (con base en el Cuadro A1 del Anexo). En tanto, el 25\% (desvío estandar 0,7) de la superficie forrajera de Uruguay se localizó en la región 2 (con base en el Cuadro A5 del Anexo). En el mismo período, el 64\% (desvío estandar 3,9) del área agrícola de Nueva Zelanda se localizó en la North Island, de esta proporción el $98 \%$ correspondió a pasturas artificiales y cultivos forrajeros y solo el $2 \%$ (desvío estándar 1) a otros cultivos (con base en fuentes del Cuadro A1 del Anexo). La región 2 de Uruguay concentró el 65\% (desvío estandar 5,5) del área destinada a la producción agrícola, y la agricultura forrajera (pasturas artificiales, campo mejorado y cultivos de forraje) representó una proporción menor, aunque creciente, del área agrícola (11\% en 1908, $20 \%$ en 1951 y $56 \%$ en 2011 , según los censos agropecuarios). 
ganaderos (carne bovina, carne ovina y lana) y permite realizar comparaciones entre establecimientos rurales y sistemas de producción (Oficialdegui, 1984). El indicador asume un costo de producción de carne y lana a partir de los requerimientos de forraje de cada especie animal, por lo que es también un buen indicador de productividad de la tierra. $\mathrm{El}$ factor de transformación aplicado tradicionalmente en Uruguay supone que la producción de un kilogramo de lana sucia requiere 2,48 veces más forraje que la producción de un kilogramo de carne (tanto vacuna como ovina) ${ }^{12}$. Esta relación fue propuesta por la CONEAT (1979) y ampliamente usada en investigaciones que han puesto el foco en el análisis del desempeño productivo de la ganadería uruguaya en el largo plazo (Astori, 1979; Becoña, 2010; Moraes, 2001). También fue aplicada en otros trabajos que compararon el desempeño productivo de la ganadería de Nueva Zelanda y Uruguay (Álvarez, 2008; Álvarez \& Bortagaray, 2007), en los que se asume, para el caso de Nueva Zelanda, el mismo factor de ponderación de uso corriente en Uruguay con base en información reportada por Monteath (1966) y por Booth (1965), que sugieren un factor de transformación lana/carne de 2,7 y 2,6, respectivamente.

A los efectos de mejorar el potencial explicativo del indicador CE, en este artículo proponemos incorporar al indicador la producción de leche, para lo cual se considera un factor de ponderación carne/leche 10:1, con base en Gómez Miller (1996: 225), y Stringleman y Scrimgeour (2012). La producción de leche ha sido un rubro de gran importancia en el sistema agrario neozelandés desde la década de 1890 y de creciente importancia en Uruguay desde mediados del siglo Xx. Adicionalmente, proponemos series alternativas del indicador CE basadas en la estimación de factores de ponderación específicos lana/carne para cada año censal, con el fin de recoger los cambios experimentados por la producción ganadera a lo largo del tiempo y atender las críticas realizadas al concepto CE.

\subsubsection{El concepto carne equivalente en discusión}

En los últimos años el indicador CE ha sido fuertemente criticado desde las ciencias agrarias. La principal crítica señala que no refleja adecuadamente los costos energéticos de la producción de carne y lana, al tiempo que asume los mismos costos energéticos de la producción de carne bovina y ovina. Aguirrezabala (1989) destaca que los costos de producción de carne bovina y ovina podrían diferir, porque no existe una única tasa de sustitución ovino-bovino debido a que las condiciones de pastoreo pueden alterar el consumo

12. La producción total en kilos de carne equivalente por hectárea en sistemas de producción ganaderos se define del siguiente modo: carne equivalente/ha $=\mathrm{kg}$ carne vacuna/ha $+\mathrm{kg}$ carne ovina/ha + (kg lana/ha x 2,48) (Olveira, 2009: 164). 
voluntario de los animales y afectar de distintas maneras el consumo de cada especie. También destaca que bovinos y ovinos tienen una serie de diferencias significativas (composición de la leche materna, ganancias de peso, efecto de las ganancias compensatorias, etc.) que determinan diferencias en el costo final en términos de requerimientos energéticos y consumo de forraje. Por lo tanto, no parece haber evidencia científica suficiente que permita estimar la equivalencia entre carne y lana. Estos son productos bioquímica, metabólica y fisiológicamente diferentes, con complejos procesos inherentes al logro de cada producto, difíciles de equiparar cuantitativamente y resumir en una única variable.

\section{CUADRO 6}

Uruguay: estimación del factor carne equivalente*

\begin{tabular}{ccccc}
\hline & $\begin{array}{c}\text { Carne bovina en pie por UG } \\
\mathbf{k g}\end{array}$ & $\begin{array}{c}\text { Carne ovina en pie por UG } \\
\mathbf{k g}\end{array}$ & $\begin{array}{c}\text { Lana por UG } \\
\mathbf{k g}\end{array}$ & Factor carne equivalente \\
\hline & $\mathbf{1}$ & $\mathbf{2}$ & $\mathbf{3}$ & $\mathbf{4 = ( 1 - 2 ) / 3}$ \\
\hline 1930 & 90,29 & 50,54 & 21,00 & 1,89 \\
1937 & 76,50 & 43,80 & 17,40 & 1,88 \\
1951 & 68,80 & 38,40 & 21,60 & 1,41 \\
1956 & 75,90 & 28,20 & 23,40 & 2,04 \\
1961 & 75,40 & 34,20 & 23,40 & 1,76 \\
1964 & 77,70 & 28,80 & 22,80 & 2,14 \\
1970 & 91,20 & 36,51 & 22,01 & 2,48 \\
1980 & 77,80 & 38,30 & 18,00 & 2,19 \\
1990 & 78,69 & 50,40 & 23,37 & 1,21 \\
2000 & 85,76 & 57,20 & 24,76 & 1,15 \\
2010 & 89,88 & 66,15 & 23,77 & 1,00 \\
\hline
\end{tabular}

*Factor carne equivalente estimado con la metodología usada por CONEAT (véase Anexo). En esta estimación, se tomaron los años correspondientes a los censos agropecuarios.

Fuentes: 1970 y 1980, factores de carne equivalente en Oficialdegui (1984: 56, 57, cuadros 1, 2, 3). 19301961 y 1990-2010, estimación propia con base en: 1930, stock animal tomado del censo agropecuario de 1930; producción de carne bovina y lana en Bértola et al. (1998), base de datos proporcionada por el autor; producción de carne ovina en Bértola (1991: 229, cuadro A.7). 1937-1964, producción de carne bovina, de carne ovina y de lana por animal en existencia en OPYPA-CIDE (1967: 323, cuadro 15; 335, cuadro 19). 1990 y 2000, stock animal tomado de los respectivos censos agropecuarios; producción de carne bovina, de carne ovina y de lana tomado de estadísticas agropecuarias y series históricas, publicadas por el Ministerio de Ganadería Agricultura y Pesca (http://www.mgap.gub.uy/). 2010, stock animal, producción de carne bovina, de carne ovina y de lana, en DIEA-MGAP (2012: 54, 55, cuadros 22, 23).

Por otra parte, Oficialdegui (1984) destaca que el indicador CE y el factor de transformación clásico (lana-carne 2,48) fue estimado por la CONEAT con base en las condiciones de la producción ganadera uruguaya del año 1970. Es sabido que el nivel de producción de un año depende de la combinación y manejo de los recursos disponibles, y 
de condiciones aleatorias, como las condiciones del tiempo (pluviosidad, temperaturas, etc.), lo que puede dar lugar a variaciones anuales importantes. Por lo tanto, si se hubiese considerado información de otros años, y no exclusivamente del año 1970, el coeficiente de ponderación lana-carne cambiaría, como muestra el Cuadro 6. La variación del coeficiente en el período $1930-2010^{13}$ es de tal magnitud que pone en dudas la robustez del factor de transformación lana-carne 2,48.

\section{CUADRO 7}

Nueva Zelanda: estimación del factor carne equivalente*

\begin{tabular}{ccccc}
\hline & $\begin{array}{c}\text { Carne bovina en pie por UG } \\
\mathbf{k g}\end{array}$ & $\begin{array}{c}\text { Carne ovina en pie por UG } \\
\mathbf{k g}\end{array}$ & $\begin{array}{c}\text { Lana por UG } \\
\mathbf{~ k g}\end{array}$ & $\begin{array}{c}\text { Factor carne equivalente } \\
\mathbf{2}\end{array}$ \\
\hline $\mathbf{1}$ & $\mathbf{2}$ & $\mathbf{3}$ & $\mathbf{4 = ( 1 - 2 ) / 3}$ \\
\hline 1931 & 47,06 & 43,76 & 24,57 & 0,13 \\
1938 & 61,66 & 47,28 & 24,95 & 0,58 \\
1951 & 80,59 & 59,99 & 31,91 & 0,65 \\
1956 & 83,90 & 49,99 & 33,24 & 1,02 \\
1961 & 63,57 & 55,65 & 32,93 & 0,24 \\
1966 & 70,38 & 53,61 & 33,69 & 0,50 \\
1971 & 68,36 & 56,39 & 32,79 & 0,36 \\
1980 & 92,75 & 48,83 & 33,24 & 1,32 \\
1990 & 101,46 & 55,38 & 32,05 & 1,44 \\
2002 & 122,81 & 81,78 & 39,02 & 1,05 \\
2010 & 154,49 & 86,79 & 32,49 & 2,08 \\
\hline
\end{tabular}

${ }^{\star}$ Factor carne equivalente estimado con la metodología usada por CONEAT (véase Anexo).

Fuentes: stock animal, fuentes de Cuadro A2 del Anexo. Carne bovina y ovina: 1931, estimado con base en cantidad y peso total de los animales sacrificados en mataderos y en los predios rurales, en SNZ (2012b); 1938-2010, en SNZ (2012b); 1939, 1958, 1959, 1963, 1972, 1981, 1992 y 2012, en SNZ (2012b). Producción de lana: 1931-1990, en Mitchell (1998) y Bloomfield (1984: 188, tabla V.16); 2000, en SNZ (2006: tabla 18.11) y SNZ (2012b); 2010, en SNZ (2010).

A los efectos de la comparación también se estimaron los coeficientes de transformación históricos de Nueva Zelanda. Para ello, se aplicó la metodología CONEAT, con base en información anual de stock animal, producción de carne ovina y bovina, y producción de lana. El Cuadro 7 muestra una importante variación del factor carne equivalente en Nueva Zelanda entre los años elegidos, incluso mayor que en Uruguay ${ }^{14}$.

13. El factor de transformación lana-carne es estimado desde 1930, porque a partir de esa fecha es posible contar con información de las variables utilizadas para el cálculo del coeficiente de una misma fuente.

14. Desviación estándar: Uruguay 0,48 y Nueva Zelanda 0,59. 
A partir de las consideraciones realizadas por Aguirrezabala (1989) y por Oficialdegui (1984), y de la información que brindan los cuadros 6 y 7, es posible afirmar que la variación del coeficiente de transformación entre los años elegidos expresa, fundamentalmente, cambios en la composición del stock y en el quantum producido en cada rubro a lo largo del tiempo, más que cambios en los requerimientos energéticos de cada especie para la producción de carne y lana.

\subsubsection{Desempeño relativo en términos de carne equivalente}

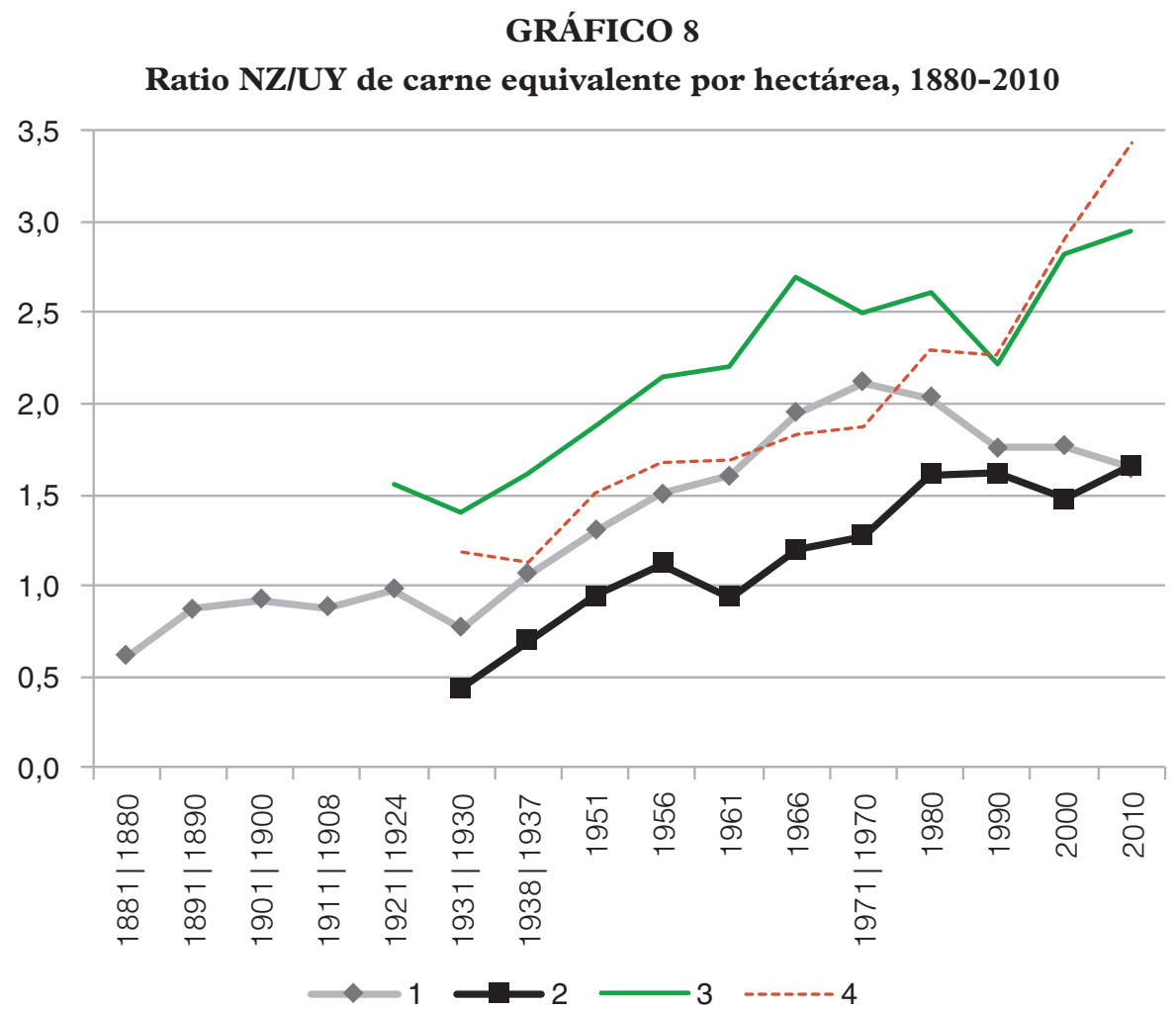

Referencias: 1. Coeficiente de ponderación clásico: carne equivalente $=\mathrm{kg}$ carne vacuna/ha $+\mathrm{kg}$ carne ovina/ha $+(\mathrm{kg}$ lana/ha $\times 2,48) .2$. Coeficiente de ponderación específico: carne equivalente $=\mathrm{kg}$ carne va$\mathrm{cuna} / \mathrm{ha}+\mathrm{kg}$ carne ovina/ha + (kg lana/ha $\mathrm{x}$ factor de ponderación en cada año). 3. Coeficiente de ponderación clásico c/leche: carne equivalente $=\mathrm{kg}$ carne vacuna/ha $+\mathrm{kg}$ carne ovina/ha $+(\mathrm{kg}$ lana/ha $\mathrm{x} 2,48)+$ (litros de leche $\mathrm{x} 0,1) .4$. Coeficiente de ponderación específico $\mathrm{c} / \mathrm{leche:}$ carne equivalente $=\mathrm{kg}$ carne vacuna/ha + kg carne ovina/ha + (kg lana/ha x factor de ponderación en cada año) + (litros de leche x 0,1). Fuentes: Nueva Zelanda, producción de carne, lana y leche (Cuadro A6); coeficientes de equivalencia (Cuadro 7), superficie de pastoreo (Cuadro A1). Uruguay, producción de carne, lana y leche (Cuadro A7), coeficientes de equivalencia (Cuadro 6), superficie de pastoreo (Cuadro A4). (véanse todos los cuadros en el Anexo). 
A los efectos de precisar la evolución del desempeño relativo de la productividad parcial de la ganadería de Nueva Zelanda y Uruguay en términos de CE por hectárea, presentamos cuatro alternativas del indicador (Gráfico 8). La tendencia que predomina es de crecimiento de la brecha a favor de Nueva Zelanda. Cada indicador muestra distintas magnitudes de la brecha, también distintos momentos en los que Nueva Zelanda se adelanta a Uruguay. Los indicadores más representativos del desempeño relativo de ambos sistemas ganaderos son los que incluyen la producción de leche, además de la de carne y lana (véanse variables 3 y 4 del Gráfico 8). En estos casos Nueva Zelanda presenta niveles mayores de productividad que Uruguay en la década de 1930 y aumenta la brecha hasta 2010.

\section{GRÁFICO 9}

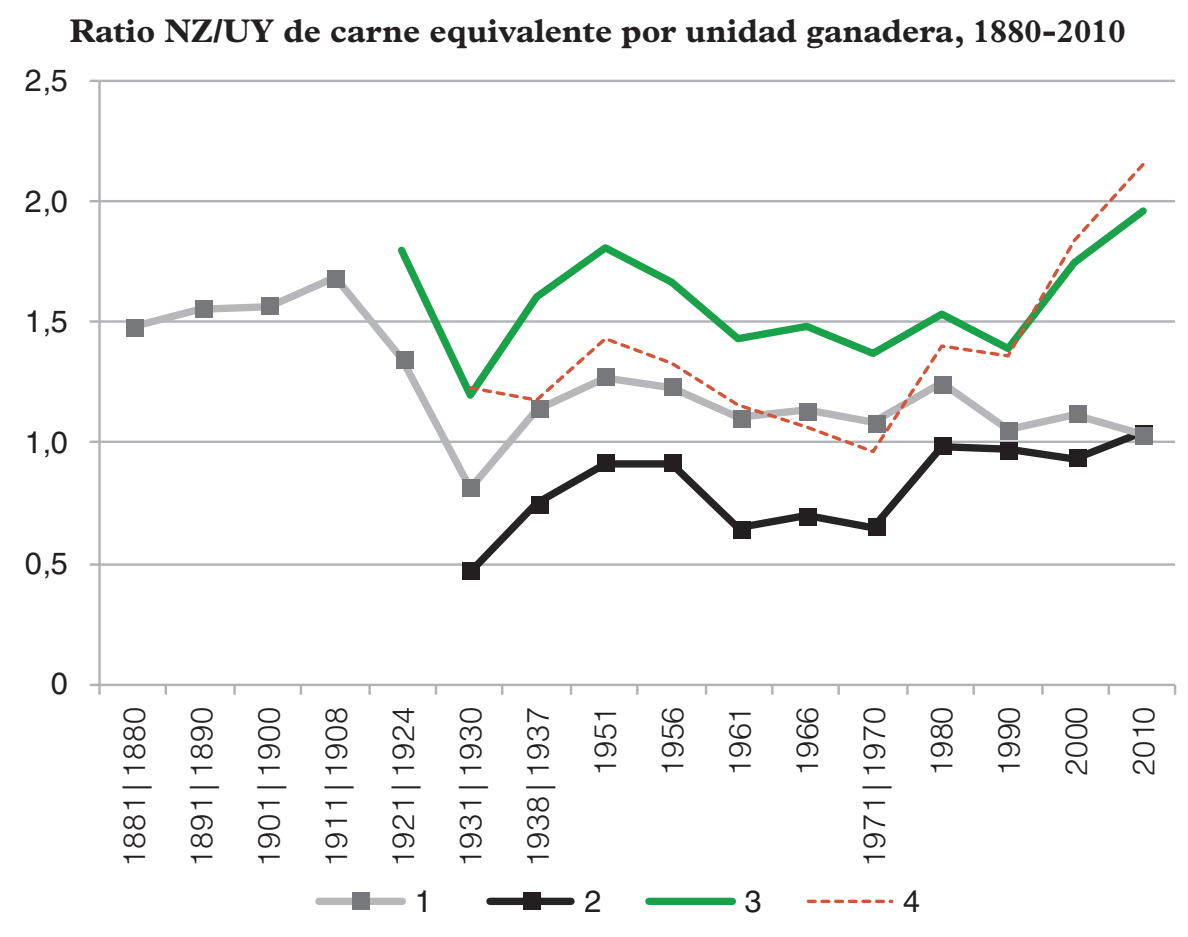

Referencias: véase Gráfico 8.

Fuentes: véanse gráficos 6 y 8 .

Otra forma de medir la evolución del desempeño relativo de la productividad física de la ganadería es considerar la relación CE por UG. El Gráfico 9 muestra niveles más altos de CE por UG en Nueva Zelanda si se tienen en cuenta los indicadores que incluyen la producción de leche. Los otros muestran una tendencia convergente, pero partiendo de distintos niveles. 
La información que brindan el Gráfico 8 y el Gráfico 9 sugiere que la divergencia de la productividad física de la ganadería entre ambos países, en términos de $\mathrm{CE}$, se debió, en mayor proporción, a la evolución de la productividad de la tierra que a la evolución de la productividad por UG. Similares tendencias se verifican si se compara el desempeño relativo de cada uno de los rubros de producción ganadera (carne bovina, carne ovina, leche y lana) por hectárea y por especie animal. Esto indica que la tasa de incorporación de tecnologías mejoradoras del factor tierra en Nueva Zelanda fue más alta que en Uruguay, más alta incluso que las mejoras en la calidad del ganado.

\section{TRAYECTORIAS TECNOLÓGICAS DE LOS SISTEMAS GANADEROS DE NUEVA ZELANDA Y URUGUAY ASOCIADAS A LA TRANSFORMACIÓN DEL FACTOR TIERRA ${ }^{15}$}

A partir de la evidencia desplegada en las secciones 2 y 3 cabe preguntarse: ¿por qué Nueva Zelanda pudo sostener más altas tasas de crecimiento de la productividad de la tierra que Uruguay en términos de UG/ha y de CE/ha? La pregunta adquiere mayor relevancia si se tiene en cuenta que la alimentación animal en Uruguay se basó en el uso de la pradera natural, en tanto en Nueva Zelanda una proporción grande y creciente de la alimentación ganadera se basó en praderas cultivadas y campos mejorados (Gráfico 10).

Para responder esta pregunta, ponemos el foco del análisis en los procesos de adopción de tecnologías mejoradoras del factor tierra y en los incentivos presentes en cada país para incorporar estas mejoras tecnológicas. El concepto central que orienta la comparación es el de trayectoria o paradigma tecnológico, desarrollado por los enfoques evolucionistas y neoschumpeterianos del cambio técnico (Lundvall, 1992; Pérez, 2009; Winter \& Nelson, 1982). Estos enfoques conciben el cambio tecnológico como un proceso tácito, acumulativo e incremental que se produce en el marco de una trayectoria, cuya difusión y adopción sigue el ritmo cambiante de una curva logística (Pérez, 2009). El enfoque es especialmente apropiado para abordar el problema del cambio tecnológico en el sector agrario, porque permite un abordaje sistémico y territorial de los procesos de innovación a través de la conceptualización de lo que Lundvall (1992), Nelson (1995) y Edquist (1997) definieron como sistemas nacionales y sectoriales de innovación.

Partiendo de estos conceptos y de la evidencia desplegada en las anteriores secciones, la hipótesis central establece que Uruguay tuvo, en el siglo XIX, mejores condiciones na-

15. Con base en Álvarez (2018). 
turales para la producción ganadera ${ }^{16}$ que Nueva Zelanda y, por tanto, menos incentivos para desarrollar tecnologías de producción de pasturas. En el caso de Nueva Zelanda las condiciones geográficas e históricas de expansión de la actividad agraria exigieron la temprana superación de una serie de problemas y restricciones, como la baja productividad de la pradera natural, principalmente en la South Island, y la necesidad de transformar el paisaje y el ecosistema nativo en la North Island, compuesto principalmente por bosques (Taylor \& Smith, 1997).

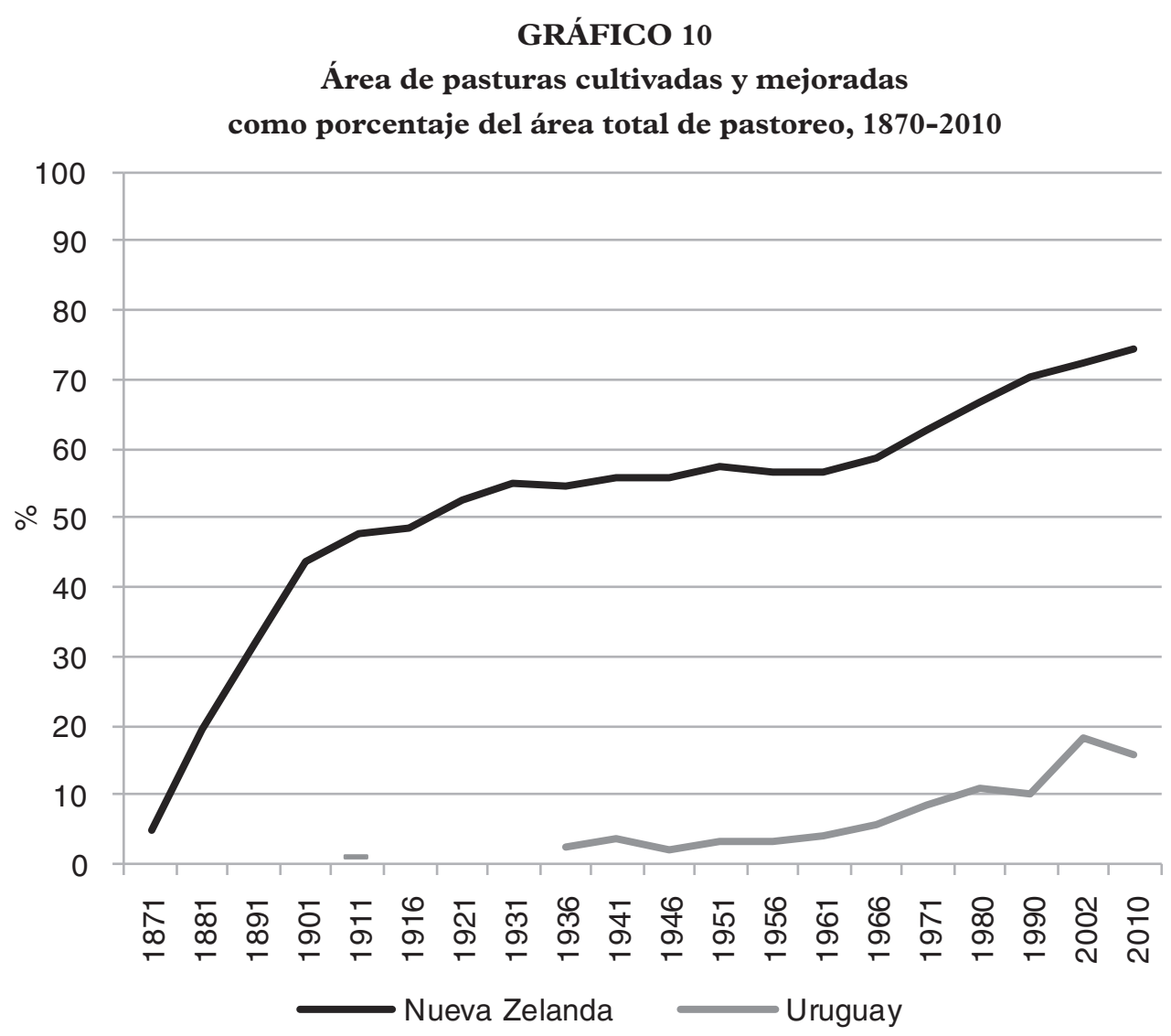

Fuentes: Nueva Zelanda (Cuadro A1 del Anexo). Uruguay (Cuadro A4 del Anexo).

Desde el punto de vista de los sistemas sectoriales de innovación, Nueva Zelanda desarrolló un enfoque sistémico del problema forrajero desde las últimas décadas del siglo XIX, creando una densa institucionalidad compuesta por universidades, centros de in-

16. Esto fue interpretado como una clase de bendición diábolica por BARRÁN y NAHUM (1978) en el sentido que SACHS y WARNER (1995) asignaron al concepto maldición de los recursos. 
vestigación, programas públicos de extensión y asociaciones de productores, en tanto Uruguay comenzó a gestar un enfoque sistémico del problema forrajero recién en la década de 1960, más allá de algunas líneas de investigación y políticas no sistémicas implementadas en las décadas anteriores (Álvarez \& Bortagaray, 2007).

Estas diferencias se vieron plasmadas en la configuración de diferentes trayectorias o paradigmas tecnológicos en ambos países a largo plazo (tres paradigmas tecnológicos en Nueva Zelanda y dos en Uruguay), asociados al incremento de la productividad de la tierra.

\subsection{Nueva Zelanda}

\subsubsection{Primer paradigma: transformación del paisaje y producción de pasturas de origen británico (1870-1920)}

En el marco de este paradigma tecnológico se completó la ocupación agraria de las planicies y praderas de la South Island y se expandió la frontera agraria en la North Island, a través de un intenso proceso de deforestación y sustitución de bosques por praderas cultivadas. El cultivo de praderas fue técnicamente menos $\operatorname{costoso}^{17}$ y más rentable que el desarrollo de otros cultivos agrícolas en las regiones deforestadas, debido a las oportunidades que generó la introducción de la refrigeración en la década de 1880 para la expansión de la ganadería lechera y la exportación de sus productos (manteca, quesos, etc.). La producción de praderas se hizo con base en la introducción de gramíneas exóticas de origen británico, lo que requirió un proceso de adaptación. El área de pasturas cultivadas creció de 0,3 a 6,7 millones de hectáreas y con ella la superficie total de pastoreo de 6,4 a 12,8 millones de hectáreas (véase Gráfico 4). Sin embargo, la técnica británica de producción de pasturas produjo el rápido agotamiento de la fertilidad natural del suelo, lo que fue compensado con la aplicación de fertilizantes químicos y orgánicos. Los altos costos y los rendimientos decrecientes de la aplicación de fertilizantes produjeron la maduración de esta trayectoria y la necesidad de profundizar el conocimiento de las deficiencias naturales del suelo para la producción de pasturas autóctonas.

17. En el período, cerca del $80 \%$ de la pastura de la North Island fue sembrada en superficie, sin roturación del suelo (yearbooks de 1901 y 1911, en SNZ, 2012a). 


\subsubsection{Segundo paradigma: desarrollo de un paquete tecnológico local de producción de pasturas (1920-1980)}

En el marco de este segundo paradigma se desarrollaron los conocimientos científicos y técnicos, relativos a plantas y suelos, que permitieron la implantación de praderas de alto rendimiento en las distintas regiones del país. Estas innovaciones consistieron en la producción de cepas perennes de ryegrass con un alto valor nutritivo para la alimentación animal y el cultivo combinado con variedades locales de trébol, que aumentaron la capacidad de fijación de nitrógeno al suelo, lo que redujo los requerimientos de fertilizantes. En una primera etapa (1920-1940), se creó un sistema de certificación de semillas que garantizó la siembra de pasturas de alta calidad en las regiones bajas y de más fácil acceso del país. En una segunda etapa (1940-1966), se difundió esta tecnología entre los productores de la South Island y de las regiones montañosas a través de la fertilización y siembra en cobertura realizada por aeroplanos. El proceso contó con nuevas inversiones en mejoras por parte de los productores e importantes inversiones en obras públicas con el fin de establecer empresas ganaderas en regiones con muy baja aptitud productiva. En la última etapa de difusión de esta tecnología (1966-1980) el área total de pastoreo creció 2,5 millones de hectáreas. Esta nueva expansión se produjo principalmente en las regiones montañosas y fue una respuesta de los productores rurales a la caída del precio de la lana en el mercado mundial. Este proceso requirió mucha investigación sobre la adaptación de las pasturas a terrenos muy inclinados y contribuyó a consolidar un sistema de manejo de pastoreo específico para estas regiones.

\subsubsection{Tercer paradigma: intensificación de la producción ganadera con sustentabilidad ecológica (1980-2010)}

Finalmente, entre 1980 y 2010 la ganadería neozelandesa ingresó en una nueva etapa de intensificación. Se redujo casi tres millones de hectáreas el área total de pastoreo y un millón de hectáreas la superficie de pasturas mejorada. Esto se debió a un conjunto de factores: la eliminación de subsidios al sector agrario, en el marco de las reformas liberales implementadas en la década de 1980 (Nightingale, 1992); un esquema de incentivos públicos para sustituir la producción ganadera en tierras marginales; y la sustitución de la producción de pasturas por la reintroducción planificada de especies vegetales nativas, especialmente forestales, en las tierras montañosas más empinadas afectadas por la erosión. Estos cambios se desarrollaron en el marco de un cambio de paradigma que colocó la sustentabilidad ecológica de la intensificación de la producción ganadera como un aspecto central del desarrollo agrario (MacLeod \& Moller, 2006) y se expresó a través de diferentes trayectorias a escala regional. Por un lado, se aplicó un modelo de transformación 
del uso del suelo con base en la biodiversidad y la sustentabilidad de la producción en las regiones altas. Por el otro, se registró un fuerte proceso de intensificación de la producción en las regiones más productivas y de más fácil acceso, especialmente asociada con la ganadería lechera (OECD, 2008). Este paradigma está aún en pleno desarrollo y búsqueda del equilibrio entre la intensificación de la producción y la sustentabilidad ecológica.

\subsection{Uruguay}

\subsubsection{Primer paradigma: producción ganadera extensiva con base en la pradera natural (1860-1950)}

La primera trayectoria tecnológica de la ganadería uruguaya se inició con el crecimiento del stock ovino en la década de 1860 y el refinamiento del ganado bovino a través de un intenso proceso de mestizaje en las décadas posteriores. El crecimiento de la producción y de la productividad fue posible por la implementación, a escala nacional, de un conjunto de transformaciones tecnológicas (cercado y alambramiento de los campos, mejoras genéticas del ganado, expansión del ferrocarril) e institucionales (fortalecimiento del poder político del Estado y consolidación de los derechos de propiedad en el medio rural) que, entre otros efectos, permitieron un uso más eficiente de la pradera natural (Barrán \& Nahum, 1977; Finch, 2005; Millot \& Bertino, 1996; Moraes, 2001). Una vez alcanzado el potencial máximo de la pradera natural para la alimentación animal, esta trayectoria ingresó en una etapa de temprana maduración hacia la Primera Guerra Mundial (Moraes, 2001, 2008), y la ganadería inició un largo período de estancamiento productivo. La principal causa del estancamiento fue no haber desarrollado ni adaptado de forma exitosa tecnologías mejoradoras del factor tierra. Entre la Primera Guerra Mundial y la década de 1950 hubo algunos intentos por atender el problema de la alimentación animal, pero ninguna solución cuajó en el desarrollo de una tecnología que permitiera aumentar o complementar la capacidad nutritiva de la pradera natural.

En la década de 1950, ante la persistencia del estancamiento productivo, hubo un viraje radical en la búsqueda de soluciones tecnológicas. Se abandonaron las líneas de investigación que procuraban mejorar la pradera natural y se importó el paquete tecnológico de producción de pasturas de Nueva Zelanda. 


\subsubsection{Segundo paradigma: importación y adopción del paquete tecnológico de producción de pasturas de Nueva Zelanda (1950-2010)}

En la década de 1950 Uruguay optó por la tecnología neozelandesa de producción de pasturas desarrollada en el marco de lo que más arriba definimos como el segundo paradigma tecnológico de Nueva Zelanda. El impacto que causó el desarrollo tecnológico de la producción ganadera neozelandesa entre los técnicos uruguayos generó una corriente favorable a su adopción que contó, además, con asistencia financiera y técnica del Banco Mundial y de la FAO (Campal, 1969; Moraes, 2001). A escala local, se crearon un conjunto de instituciones con participación del sector público y de los productores, y se conformaron centros de investigación agronómica y estaciones experimentales para adaptar la tecnología neozelandesa (Álvarez \& Bortagaray, 2007; Astori, 1979). Si bien el área con praderas cultivadas y mejoradas se triplicó en Uruguay entre 1956 y 1990, pasando de 476.000 hectáreas a 1,5 millones de hectáreas, los resultados fueron escasos, tanto en términos del área abarcada, que apenas alcancó el $10 \%$ del área total de pastoreo (véase Gráfico 10), como en términos del crecimiento de la producción. La limitada difusión de esta tecnología se debió al alto riesgo que supuso para los productores la inversión en pasturas por el escaso conocimiento agronómico acumulado en Uruguay para la aplicación de esta tecnología (Astori, 1979; Paolino, 1990; Reig \& Vigorito, 1986) y por la baja rentabilidad de la inversión en pasturas artificiales (Instituto de Economía, 1969; Jarvis, 1982).

No obstante estos modestos resultados, la difusión de la tecnología impactó de distintas formas a escala regional (Paolino, Sosa \& Durán, 1987). Las regiones de Uruguay con tierras más productivas y con una especialización agrícola, agrícola-ganadera y lechera -que coinciden con la región 2 (véase Sección 3.3)- mostraron una mayor tasa de adopción de la nueva tecnología, especialmente en la producción de praderas artificiales, modalidad que requería una acumulación previa de prácticas y conocimientos agrícolas. La región típicamente ganadera, orientada principalmente a la producción de carne y lana (región 1), mostró tasas de adopción más bajas y las técnicas de mejoramientos adoptadas fueron de tipo extensivo (fertilización y siembra en cobertura en campo natural) (Paolino, 1990). Estas trayectorias regionales continuaron luego de la década de 1990 cuando comenzó a superarse el largo estancamiento de la producción ganadera que se había iniciado en las primeras décadas del siglo xx (Buxedas, 2001; Mondelli \& Picasso, 2001).

El cambio de tendencia que permitió superar el largo estancamiento productivo fue el resultado del conocimiento acumulado desde la década de 1950. Luego de un largo período de aprendizaje, mejoró la eficiencia productiva y económica de la inversión en praderas artificiales, y se verificó una coyuntura nacional e internacional propicia para la inversión en la ganadería. 
Entre 1990 y 2000 la superficie de campos mejorados, praderas artificiales y cultivos forrajeros creció de 1,5 a 2,4 millones de hectáreas, al tiempo que se incrementó la cantidad promedio de potreros por establecimiento. La combinación de estos cambios permitió introducir mejoras en el manejo animal, como la reducción de la edad de faena y el aumento de la tasas de extracción, y verificar también distintas trayectorias a escala regional. Finalmente, en la década 2000-2010 la ganadería mantuvo y profundizó la dinámica de intensificación de la producción, especialmente la ganadería bovina de carne y leche. La incorporación de otras técnicas de alimentación animal, basadas en el uso creciente de alimentos concentrados, permitió el crecimiento de la producción de carne y leche, sin variaciones significativas de la carga animal por hectárea.

\section{CONSIDERACIONES FINALES}

La actividad ganadera ha sido clave en las historias económicas de Nueva Zelanda y Uruguay desde el siglo XIX hasta la actualidad, lo que ha determinado el patrón de especialización productiva y de inserción en la economía mundial, y, a través de diversos canales, el desempeño a largo plazo de ambas economías.

Las dos principales contribuciones de este artículo han sido identificar las principales tendencias relativas de la productividad física de la ganadería de ambos países a escala nacional y regional, y destacar la importancia que tuvo el crecimiento de la productividad de la tierra en el desempeño a largo plazo del sector. Para ello, se realizó una revisión de las bases conceptuales de los indicadores sintéticos de productividad parcial como la UG y la CE, y se identificaron distintas trayectorias tecnológicas vinculadas a la producción de pasturas.

Los principales resultados indican que ambos países experimentaron una suerte de reversión de las condiciones de partida: en las últimas décadas del siglo XIX, la productividad de la tierra de uso ganadero en Uruguay, en términos de UG/ha y CE/ha, fue más alta, casi el doble, que la de Nueva Zelanda; sin embargo, la ganadería neozelandesa mostró ritmos de crecimiento más altos a largo plazo hasta duplicar, e incluso triplicar, los niveles de Uruguay en la segunda mitad del siglo xx.

Estas trayectorias divergentes fueron el resultado de los desafíos que enfrentó cada sistema ganadero para incrementar la producción. En tanto Uruguay contó con condiciones naturales muy favorables para la producción ganadera y no requirió incorporar mejoras a la tierra hasta las primeras décadas del siglo Xx, Nueva Zelanda, desde el siglo XIX, tuvo que modificar el paisaje nativo, desarrollar tecnologías de implantación de pasturas 
artificiales y transformar el uso del suelo para aumentar y diversificar la producción ganadera. Para ello, Nueva Zelanda transitó por distintas trayectorias tecnológicas que fueron mejorando y refinando las técnicas de producción de pasturas. El primer paradigma tecnológico -en el que se adaptaron pasturas y técnicas importadas de Gran Bretaña- y el segundo paradigma -que produjo variedades y técnicas locales-fueron desarrollados para hacer posible la producción ganadera de alto rendimiento en la North Island. La difusión de esta tecnología a regiones menos productivas del país tomó varias décadas de ensayos y aprendizajes, lo que explica las grandes diferencias que muestran la North Island y la South Island en términos de UG/ha. Uruguay, luego de haber agotado una primera trayectoria tecnológica, que alcanzó el potencial máximo de la pradera natural hacia 1914, y de haber atravesado varias décadas de estancamiento productivo, importó en la década de 1950 el paquete tecnológico de producción de pasturas neozelandés. Uruguay requirió varios años de aprendizaje para adaptar la tecnología de forma eficiente y su adopción estuvo limitada a ciertas regiones del territorio. Por tratarse de una tecnología que demandaba suelos con buena aptitud agrícola y cierta experiencia de los productores para el manejo combinado de agricultura y ganadería, la técnica se adaptó y adoptó en mayor medida en la región suroeste del país (región 2), e impactó más en la ganadería lechera que en la de carne y lana.

\section{AGRADECIMIENTOS}

Una versión preliminar de este artículo fue presentada en el XVI Congreso de Historia Agraria, SEHA, Santiago de Compostela, 20-23 junio de 2018, sesión «Quantitative Agricultural History: institutions, markets and natural resources». Agradezco a los organizadores de la sesión y los comentarios recibidos en esa ocasión. También a la Comisión Sectorial de Investigación Científica de la Universidad de la República, Uruguay, y a «Stout Research Centre for New Zealand Studies, Victoria University of Wellington» por el apoyo brindado. Un especial agradecimiento al profesor Luis Bértola por su orientación y apoyo permanente y a los evaluadores anónimos de Historia Agraria por los valiosos comentarios y sugerencias realizados.

\section{REFERENCIAS}

Aguirrezabala, M. (1989). Modelo de simulación del consumo de ovinos y bovinos en condiciones de pastoreo: Análisis de componentes y sintesis del modelo. Tesis de grado. Montevideo: Universidad de la República. 
Aguirrezabala, M. \& Oficialdegui, R. (1995). Experimentación simulada del efecto de la época de apareamiento de ovinos y bovinos sobre el consumo de forraje y la capacidad de carga. Producción Ovina SUL, (7), 23-34.

ÁlvareZ, J. (2007). Nueva Zelanda y Uruguay: Una renovada tradición de estudios comparados. En J. Álvarez, L. BérTola \& G. PorCile (Eds.), Primos ricos y empobrecidos: Crecimiento, distribución del ingreso e instituciones en Australia-Nueva Zelanda vs Argentina-Uruguay (pp. 191-208). Montevideo: Fin de Siglo.

Álvarez, J. (2008). Instituciones, cambio tecnológico y distribución del ingreso: Una comparación del desempeño económico de Nueva Zelanda y Uruguay (1870-1940). Tesis de maestría. Montevideo: Universidad de la República.

ÁlvareZ, J. (2018). Technological Change and Productivity Growth in the Agrarian Systems of New Zealand and Uruguay (1870-2010).En V. Pinilla \& H. Willebald (Eds.), Agricultural Development in the World Periphery. A Global Economic History Approach (pp. 467-492). Cham: Palgrave Macmillan. https://doi.org/10.1007/978-3319-66020-2

ÁlvareZ, J. (2014). Instituciones, cambio tecnológico y productividad en los sistemas agrarios de Nueva Zelanda y Uruguay. Patrones y trayectorias de largo plazo (1870-2010). Tesis de Doctorado. Programa de Historia Económica y Social, Universidad de la República, Montevideo.

Álvarez, J. \& BÉRTOLA, L. (2013). So Similar, So Different: New Zealand and Uruguay in the World Economy. En C. Lloyd, J. Metzer \& R. Sutch (Eds.), Settler Economies in World History (pp. 493-520). Leiden: Brill.

ÁlvareZ, J. \& BorTAGARAY, I. (2007). El marco institucional de la innovación agropecuaria en Nueva Zelanda y Uruguay en el largo plazo. En J. Álvarez, L. BÉRTOLA \& G. PORCILE (Eds.), Primos ricos y empobrecidos: Crecimiento, distribución del ingreso e instituciones en Australia-Nueva Zelanda vs Argentina-Uruguay (pp. 233-272). Montevideo: Fin de Siglo.

Aparicio, G., González Esteban, A. L., Pinilla, V. \& Serrano, R. (2018). The World Periphery in Global Agricultural and Food Trade, 1900-2000. En Agricultural Development in the World Periphery (pp. 63-88). Cham: Springer International Publishing. ARdente, A., Díaz, F. \& Rossi, T. (2004). Crecimiento económico y distribución del ingreso en el Uruguay. Documentos de Trabajo-Instituto de Economía, (10/04).

Astori, D. (1979). La evolución tecnológica de la ganadería uruguaya 1930-1977. Montevideo: Ediciones de la Banda Oriental.

Banco Central del Uruguay (BCU) (1973). Boletín estadístico mensual. Montevideo: Banco Central del Uruguay.

Banco Central del Uruguay (BCU) (1979). Boletín estadístico mensual. Montevideo: Banco Central del Uruguay. 
Banco de la República Oriental del Uruguay (BROU) (1965). Cuentas nacionales. Montevideo: Departamento de Investigaciones Económicas.

BARRÁn, J. P. \& NAHUm, B. (1977). Historia rural del Uruguay moderno: 6. La civilización ganadera bajo Batlle, 1905-1914. Montevideo: Ediciones de la Banda Oriental.

Barrán, J. P. \& Nahum, B. B. (1978). Historia rural del Uruguay moderno: 7. Agricultura, crédito y transporte bajo Batlle (1905-1914). Montevideo: Ediciones de la Banda Oriental.

BeCoÑA, G. (2010). Comparación del impacto ambiental en relación a gases de efecto invernadero en sistemas ganaderos de Uruguay y Nueva Zelanda. Agresearch, (2).

Bertino, M. \& TAJAm, H. (1999). El PBI de Uruguay: 1900-1955. Montevideo, Universidad de La República, Instituto de Economía.

BÉRTOLA, L. (1991). La industria manufacturera uruguaya 1913-1961: Un enfoque sectorial de su crecimiento, fluctuaciones y crisis. Montevideo: CIEDUR.

BÉrTolA, L. (2005). A 50 años de la curva de Kuznets: Crecimiento económico y distribución del ingreso en Uruguay y otras economías de nuevo asentamiento desde 1870. Investigaciones de Historia Económica, 1 (3), 135-176.

BÉRTOLA, L. (2016). El PBI per capita de Uruguay 1870-2015: Una reconstrucción. Progama de Historia Económica y Social. Documentos de Trabajo, (48).

Bértola, L., Calicchio, L., Camou, M. M. \& Rivero, L. (1998). El PBI uruguayo: 1870-1936 y otras estimaciones. Montevideo: Universidad de la República, Facultad de Ciencias Sociales.

Bertoni, R. \& Willebald, H. (2016). Do Natural Energy Endowments Matter?: New Zealand and Uruguay in a Comparative Perspective, 1870-1940. Australian Economic History Review, 56 (1), 70-99.

Bertram, G. (2009). The New Zealand Economy:1900-2000. En G. Byrnes (Ed.), The New Oxford History of New Zealand. Melbourne: Oxford University Press.

Bloomfield, G. T. (1984). New Zealand: A Handbook of Historical Statistics. Boston: G. K. Hall.

Boné, G. \& Perugorría, A. (Eds.) (2011). Manejo del rodeo de cría sobre campo natural. Montevideo: Plan Agropecuario.

Bonino, N., RomÁn, C. \& Willebald, H. (2012). PBI y estructura productiva en Uruguay (1870-2011): Revisión de series históricas y discusión metodológica. Documentos de Trabajo-Instituto de Economía, (05/12).

Bonino-Gayoso, N., Tena-Junguito, A. \& Willebald, H. (2015a). Uruguay and the First Globalization: On the Accuracy of Export Performance, 1870-1913. Revista de Historia Económica-fournal of Iberian and Latin American Economic History, 33 (2), 287-320. 
Bonino-Gayoso, N., Tena-Junguito, A. \& Willebald, H. (2015b). Uruguay and the First Globalization: On the Accuracy of Export Performance, 1870-1913. Documentos de Trabajo-Instituto de Economía, (02/2015).

Bоoтн, A. K. (1965). Crops and Pasture in the Rangitikei District. NZ Grassland Association, (27), 75-82.

Briggs, P. (2003). Looking at the Numbers: A View of New Zealand's Economic History. Wellington: NZ Institute of Economic Research.

Brooking, T. \& Pawson, E. (2011). Seeds of Empire:The Environmental Transformation of New Zealand. New York: I. B.Tauris.

BuXEDAs, M. (2001). El Uruguay ganadero: Del auge a la aftosa. Montevideo: CIEDUR. (Seminarios y talleres, 120).

Campal, E. F. (1969). La pradera. Montevideo: Nuestra Tierra. (Nuestra tierra, 28).

Census and Statistiscs Office (CSO) (1929). New Zealand Official Yearbook. Wellington: Govt. Print. https://www3.stats.govt.nz/New_Zealand_Official_Yearbooks/1929/NZOYB_ 1929.html

CLAEH-CINAM (1963). Situación económica y social del Uruguay rural. Montevideo: Ministerio de Ganadería y Agricultura.

CONEAT (1979). Grupos de suelos CONEAT: Índice de productividad. Montevideo: Comisión Nacional de Estudio Agronómico de la Tierra/Ministerio de Ganadería, Agricultura y Pesca.

Coop, I. (1965). A Review of the Ewe Equivalent System. New Zealand Agriculture Science, 1 (3), 13-18.

CoRnForth, I. S. \& SinClair, A. G. (1984). Fertiliser Recommendations for Pasture and Crops in Nerw Zealand. 2. ${ }^{a}$ rev. Wellington: Ministry of Agriculture \& Fisheries.

CREMPIEN, C. (1982). Antecedentes técnicos y metodología básica para utilizar en presupuestación en establecimientos ganaderos: Bovinos para carne y ovinos. Montevideo: Editorial Agropecuaria Hemisferio Sur.

CURRY, L. (1963). Regional Variation in the Seasonal Programming of Livestock Farms in New Zealand. Economic Geography, 39 (2), 95-118.

Dalton, C. (2009). Sheep Farm Husbandry: Practical Feeding Advice. http://woolshed1.blogspot.com/

Davie, F. D. (1960). El ejemplo de Nueva Zelanda. Montevideo: Ed. Juan A. Peri.

Denoon, D. (1983). Settler Capitalism: The Dynamics of Dependent Development in the Southern Hemisphere. Oxford: Clarendon University Press.

DIEA (2015). Regiones agropecuarias del Uruguay. Montevideo: Ministerio de Ganadería, Agricultura y Pesca. http://www.mgap.gub.uy/dieaanterior/regiones/Regiones2015.pdf DIEA-MGAP (2012). Anuario estadístico agropecuario. Montevideo: Ministerio de Ganadería, Agricultura y Pesca. http://www.mgap.gub.uy 
EDQuIST, C. (1997). Systems of Innovation:Technologies, Institutions, and Organizations. London: Pinter.

EDwARDS, S. \& Holmes, F. (1994). CER: Economic Trends and Linkages. Wellington: National Bank of New Zealand/Institute of Policy Studies.

Facultad de Ciencias Sociales, Universidad de la República (FCS) (2013). Banco de datos exportaciones, clasificación por principales secciones de la NADE. http://cienciassociales.edu.uy/bancosdedatos/seccionesarea-de-informacion-socio-demografica/series-economicas/comercio-exterior-2/

FawCEtt, E. J. \& Paton, W. N. (1929). Livestock Production: A Review of Livestock Production in New Zealand during the Past 26 Seasons (1901/2 to 1926/27), Based on Standard Values and Units. The New ZealandYear-Book, (37), 990-1000.

FINCH, M. H. J. (2005). La economía politica del Uruguay contemporáneo, 1870-2000. Montevideo: Ediciones de la Banda Oriental.

Fleming, P. H. (Ed.) (2003). Farm Technical Manual. Christchurch: Lincoln University/Farm Management Group. http://researcharchive.lincoln.ac.nz/handle/10182/5625

Gallinal, A. (1951). Enseñanzas de un rápido viaje a Nueva Zelanda. Montevideo: Florensa \& Lafon.

Gómez Miller, R. (1996). Lechería, entre lo real y lo posible. En Almanaque del Banco de Seguros del Estado 96. Montevideo: Banco de Seguros del Estado.

Instituto de EConomía (1969). El proceso económico del Uruguay. Montevideo: Universidad de la República.

INSTITUTO NACiONAL DE Estadística (INE) (2013).Valor de las exportaciones por año. http://www.ine.gub.uy/web/guest/comercio-internacional-y-balanza-de-pagos

Instituto Nacional de Investigación Agropecuaria (INIA) (2012). Revisión y análisis de las bases históricas y científicas del uso de la equivalencia ovino-bovino: $\mathrm{Ha}$ cia una nueva equivalencia para ser utilizada en Uruguay. Montevideo: INIA.

JARVIS, L. (1982). Tercer y cuarto proyecto del Banco Mundial para desarrollar el sector ganadero en Uruguay. En A. PÉrez, G. J. SECCo \& L. Jarvis (Eds.), La ganadería de carne vacuna en el Uruguay. Cali: Centro Internacional de Agricultura Tropical.

KIRBY, J. (1975). On the Viability of Small Countries: Uruguay and New Zealand Compared. Fournal of Interamerican Studies and World Affairs, 17 (3).

LANFRANCO, B. \& SAPRIZA, G. (2011). El índice CONEAT como medida de productividad y valor de la tierra. Seria Técnica INIA, (187).

LEWIS, W.A. (1983). Crecimiento y fluctuaciones, 1870-1913. México, DF: Fondo de Cultura Economica.

LineHAM, B.T. (1968). New Zealand's Gross Domestic Product, 1918/38. New Zealand Economic Papers, 2 (2), 16-26.

Lloyd, C., Metzer, J. \& Sutch, R. (2013). Settler Economies in World History. Leiden/Boston: Brill. (Global Economic History Series, 9). 
LuNDVALL, B. (1992). National Systems of Innovation:Toward a Theory of Innovation and Interactive Learning. London: Pinter.

MACLEOD, C. J. \& Moller, H. (2006). Intensification and Diversification of New Zealand Agriculture since 1960: An Evaluation of Current Indicators of Land Use Change. Agriculture, Ecosystems \& Environment, 115 (1-4), 201-218.

MAdDison, A. (2009). Historical Statistics of the World Economy: 1-2008 AD. http://www.ggdc.net/maddison/Historical_Statistics/horizontal-file_02-2010.xls

Maddison-Project (2013). The Maddison Project Database. http://www.ggdc.net/maddison/maddison-project/home.htm

Millot, J. \& Bertino, M. (1996). Historia económica del Uruguay (1860-1910). Montevideo: Fundación de Cultura Universitaria.

Mrtchell, B. R. (1998). International Historical Statistics: Africa, Asia E̋ Oceania 17501993. 3. ${ }^{\mathrm{a}}$ ed. Basingstoke: Macmillan.

Mondelli, M. \& Picasso, V. (2001). Trayectorias tecnológicas en la ganadería uruguaya: Un enfoque evolucionista. Tesis de grado. Montevideo: Universidad de la República.

Monteath, M.A. (1966). Vacuum Silage: An Aid to More Efficient Meat and Wool Production in West Otago and Southland. NZ Grassland Association, (28), 147-153.

Moraes, M. I. (2001). Las determinantes tecnológicas e institucionales del desempeño ganadero en el largo plazo, 1870-1970. Tesis de maestría. Montevideo: Universidad de la República.

Moraes, M. I. (2008). La pradera perdida: Historia y economía del agro uruguayo: Una visión de largo plazo, 1760-1970. Montevideo: Linardi y Risso.

NeLson, R. R. (1995). Recent Evolutionary Theorizing About Economic Change. Fournal of Economic Literature, 33 (1), 48-90.

Nightingale, T. (1992). White Collars and Gumboots: A History of the Ministry of Agriculture and Fisheries, 1892-1992. Palmerston North: Dunmore Press.

Oficialdegui, R. (1984). Carne equivalente: Los riesgos de la simplificación. Boletín Técnico. SUL, (13), 55-62.

Oficina de Programaciony Politica Agropecuaria-Comision de Inversionesy DeSARROLlo ECONOMICO (OPYPA-CIDE) (1967). Estudio económico y social de la agricultura en el Uruguay. Tomos I y II. Montevideo: Ministerio de Ganadería y Agricultura.

Olveira, G. (2009). Indicadores utilizados para el análisis y diagnóstico de sistemas de producción ganaderos. En J. Álvarez \& O. FAlCAO (Eds.), Manual de gestión de empresas agropecuarias (pp. 89-96). Montevideo: Universidad de la República.

ORGANISATION FOR ECONOMIC CO-OPERATION AND DEVELOPMENT (OECD) (2008). Environmental Performance of Agriculture in OECD countries since 1990. New Zealand Country Section. Paris: OECD.

PAolino, C. (1990). Estagnacao e dinamismo na agropecuaria uruguaia: Uma aborda- 
gem heterodoxa. Tesis de doctorado. Sao Paulo: Universidade Estadual de Campinas. Paolino, C., Sosa, S. \& Durán, H. (1987). El proceso tecnológico en la producción de leche. En C. BARBATo \& C. PAOlino (Eds.), Una década de cambio en la lechería uruguaya (1975-1985). Montevideo: Centro de Investigaciones Economicas/Ediciones de la Banda Oriental.

PARKER, W. J. (1998). Standardisation between Livestock Classes: The Use and Misuse of the Stock Unit System. Proceedings of the New Zealand Grassland Association, (60), 243-248.

PÉrEZ, C. (2009). Technological Revolutions and Techno-Economic Paradigms. Working Papers in Technology Governance and Economic Dynamics, (20).

Philpott, B. P. \& Hussey, D. D. (1969). Productivity and Income of New Zealand Agriculture, 1921-67. Agricultural Economics Research Unit, (59).

REIG, N. \& Vigorito, R. (1986). Excedente ganadero y renta de la tierra: Uruguay 19301970. Montevideo: Centro de Investigaciones Economicas/Ediciones de la Banda Oriental.

SACHS, J. D. \& Warner, A. M. (1995). Natural Resource Abundance and Economic Ggrowth. National Bureau of Economic Research Working Paper, (5398).

SCHLÜTER, A. (2014). Institutions and Small Settler Economies: A Comparative Study of New Zealand and Uruguay, 1870-2008. New York: Palgrave Macmillan.

Statistics New Zealand (SNZ) (2006). New Zealand OfficialYearbook. Wellington: Statistics New Zealand. https://www3.stats.govt.nz/New_Zealand_Official_Yearbooks/2006/NZOYB_2006.html?_ga=2.196352620.1997269711.15645626951308512976.1564561995

Statistics New Zealand (SNZ) (2010). New Zealand OfficialYearbook. Wellington: Statistics New Zealand. http://archive.stats.govt.nz/browse_for_stats/snapshots-of-nz/digital-yearbook-collection/2010-yearbook.aspx

Statistics New ZEALAND (SNZ) (2011). National Accounts (Industry Benchmarks):Year Ended March 2011. http://www.stats.govt.nz/browse_for_stats/economic_indicators/NationalAccounts/NationalAccountsIndustryBenchmarks_HOTPYeMar11/Com mentary.aspx

Statistics New Zealand (SNZ) (2012a). New Zealand Official Yearbook. Wellington: Statistics New Zealand. http://www.stats.govt.nz/browse_for_stats/snapshots-of-nz/yearbook.aspx

StATiSTiCs New ZeALAND (SNZ) (2012b). New Zealand Yearbook collection: 1893-2012. http://www.stats.govt.nz/yearbooks

Stringleman, H. \& Scrimgeour, F. (2012). Dairying and Dairy Products: On the Farm. En Te Ara-the Encyclopedia of New Zealand (https:/teara.govt.nz/en).

TAYLOR, R. \& SMITH, I. (1997). The State of New Zealand's Environment. Wellington: Ministry for the Environment. 
Tennant, D. B. (1978). Soil-Amending Technology, Grassland Farming, and Nerw Zealand Economic Development. Tesis de doctorado. Auckland: Massey University. Winter, S. G. \& Nelson, R. R. (1982). An Evolutionary Theory of Economic Change. Cambridge: The Belknap Press of Harvard University Press.

Woodford, K. \& Nicol, A. A. (2004). A Re-Assessment of the Stock Unit System. Ministry of Agriculture and Forestry (MAF) Information Paper, (2005/02).

WorLD BANK (2014). World Development Indicators. Washington: The World Bank. http://data.worldbank.org/data-catalog/world-development-indicators/wdi-2014 